\title{
The Complete Cloroplast Genomes of Tetrastigma Hemsleyanum (Vitaceae) from Different Regions of China: Molecular Structure, Comparative Analysis and Development of DNA Barcodes for its Geographical Origin Discrimination
}

\author{
Shujie Dong \\ School of Pharmaceutical Sciences, Zhejiang Chinese Medical University \\ Manjia Zhou \\ School of Pharmaceutical Sciences, Zhejiang Chinese Medical University \\ Jinxing Zhu \\ Bureau of Agricultural and Rural Affairs of Suichang, Suichang, China \\ Qirui Wang \\ School of Pharmaceutical Sciences, Zhejiang Chinese Medical University \\ Yuqing Ge \\ The First Affiliated Hospital of Zhejiang Chinese Medical University, Hangzhou, China \\ Rubin Cheng ( $\nabla$ rubincheng@zcmu.edu.cn) \\ School of Pharmaceutical Sciences, Zhejiang Chinese Medical University
}

Research Article

Keywords: Tetrastigma hemsleyanum, chloroplast genome, phylogenetic relationships, nucleotide diversity, DNA barcoding markers, geographical origins.

Posted Date: March 22nd, 2022

DOI: https://doi.org/10.21203/rs.3.rs-1308872/v2

License: (1) This work is licensed under a Creative Commons Attribution 4.0 International License. Read Full License 


\section{Abstract}

Background Tetrastigma hemsleyanum is a valuable traditional Chinese medicinal plant widely distributed in the subtropical areas of China. It belongs to the Cayratieae tribe, family Vitaceae, and exhibited significant anti-tumor and anti-inflammatory activities. However, obvious differences were observed on the quality of $T$. hemsleyanum root from different regions, requiring the discrimination strategy for the geographical origins.

ResultThis study characterized five complete chloroplast (cp) genomes of $T$. hemsleynum samples from different regions, and conducted a comparative analysis with other representing species from family Vitaceae to reveal the structural variations, informative markers and phylogenetic relationships. The sequenced $\mathrm{cp}$ genomes of $T$. hemsleyanum exhibited a conserved quadripartite structure with full length ranging from 160,124 bp of Jiangxi Province to 160,618 bp of Zhejiang Province. We identified 112 unique genes (80 protein-coding, 28 tRNA and 4 rRNA genes) in the cp genomes of $T$. hemsleyanum with highly similar gene order, content and structure. The IR contraction/expansion events occurred on the junctions of $y c f 1, r p s 19$ and rp/2 genes with different degrees, causing the differences of genome sizes in T. hemsleyanum and Vitaceae plants. The number of SSR markers discovered in T. hemsleyanum was 56-57, exhibiting multiple differences among the five geographic groups. Phylogenetic analysis based on conserved cp genome proteins strongly grouped the five $T$. hemsleyanum species into one clade, showing a sister relationship with $T$. planicaule. Comparative analysis of the cp genomes from $T$. hemsleyanum and Vitaceae revealed five highly variable spacers, including 4 intergenic regions and one protein-coding gene (ycf1). Furthermore, five mutational hotspots were observed among $T$. hemsleyanum cp genomes from different regions, providing data for designing DNA barcodes $\operatorname{trn} L$ and $\operatorname{trnN}$. The combination of molecular markers of $\operatorname{trn} L$ and $\operatorname{trnN} \mathrm{N}$ clustered the $T$. hemsleyanum samples from different regions into threegroups, thus successfully separating specimens of Sichuan and Zhejiang from other areas.

Conclusion Our study obtained the chloroplast genomes of $T$. hemsleyanum from different regions, andprovided a potential molecular tracing tool for determining the geographical origins of $T$. hemsleyanum, as well as important insights into the molecular identification approach and and phylogeny in Tetrastigma genus and Vitaceae family.

\section{Introduction}

Tetrastigma hemsleyanum Diels et Gilg ( $T$. hemsleyanum) is a unique and valuable Chinese medicinal herb belonging to the tribe cayratieae of family Vitaceae. It is mainly distributed around the central, eastern, southern and southwestern provinces of China [1]. As a customary Chinese medicine, $T$. hemsleyanum has been recorded in the Zhejiang Provincial Standards of Processing Chinese Crud Drugs (2015) with the therapeutic effects of heatclearing, toxicity-removing, promoting blood circulation and pain relief. The roots of $T$. hemsleyanum have been used traditionally to treat high fever, pneumonia, hepatitis, and multiple types of cancers alone or in combination with other herbal medicines [2]. Recent pharmacological investigations have demonstrated that $T$. hemsleyanum possesses anti-inflammatory, anti-virus, anti-tumor and immunomodulatory effects, which could be attributed to the active components of flavonoids, polysaccharides, terpenoids and alkaloids [3]. Total flavones of $T$. hemsleyanum could inhibit the proliferation and induce apoptosis of breast cancer cells MDA-MB-468 and MCF-7 by inhibiting the expression of p-p42/44 and blocking MAPK signaling pathway [4]. In addition, dietary flavone from $T$. hemsleyanum vine was also found to trigger human lung adenocarcinoma apoptosis via autophagy [5]. The extracts from T. hemsleyanum leaves exhibited protective effects against acrylamide induced toxicity both in HepG2 cells and Caenorhabditis elegans via regulating DAF-16/FOXO signaling pathway [6]. Furthermore, the polysaccharide isolated from $T$. hemsleyanum enhanced the immune responses in both OVA-immunized C57BL/6 mice and Lewis lung carcinoma bearing mice through activating TLR4 signaling pathway [7]. The phenolic contents of T. hemsleyanum leaves exhibited significant antioxidant activities, indicating the possibility of developing T. hemsleyanum leaves as functional foods [8]. Moreover, the herbal formula Hua Shi Xuan Fei mixture mainly composed of $T$. hemsleyanum has been approved by Zhejiang Provincial Drug Administration for clinical treatment of COVID-19, which played important roles in fighting the epidemic [9]. The high medicinal and economic value of $T$. hemsleyanum makes its further genetic and phylogenetic investigation necessary.

The roots of $T$. hemsleyanum were one of the most popular Chinese folk medicines in herbal market with the common name Sanyeqing. The genus Tetrastigma contained more than 100 species of climbers basically distributed in the tropics and subtropics of Asia, and a few species extending to Australia [10]. The Tetrastigma climbers have also attracted a ton of of attention due to its unique host-parasite association with Rafflesiaceae, which possess the largest flowers in the world [11]. The great number of Tetrastigma species provided rich resources of root tubers for the potential counterfeits for T. hemsleyanum. Due to the lack of typical identification characteristics, it is difficult to distinguish roots of $T$. hemsleyanum from those adulterants of climbers in the genus of Tetrastigma and other closely related species. The increased number of counterfeit products and substitutes of $T$. hemsleyanum in the herbal medicine market have seriously harmed the clinical safety and effectiveness of $T$. hemsleyanum and significantly threatened its healthy development [12]. For instance, the root tubers of toxic Aconitum carmichaeli have been often sold as adulterants of T. hemsleyanum in medicine markets owing to high profit in Zhejiang Province, which caused many serious poisoning incidents. Furthermore, significant differences have been indicated in chemical compositions and therapeutic values of medicinal plants from different regions, which could be attributed to genetic varieties and growth environment [13]. T. hemsleyanum was widely distributed in the tropical to subtropical areas of China with multiple varieties and large gaps in the yield and quality of root tubers. The molecular investigations of T. hemsleyanum based on ISSR and SRAP analysis revealed the high genetic diversity at the level of species and low diversity in populations [14]. The T. hemsleyanum samples from Zhejiang and Fujian Province exhibited the highest contents of total flavonoids and strongest inhibitory activities against HepG2 cells, further confirming the curtail role of the geographical origins [15]. Numerous different expressed transcripts and multiple differentially accumulated metabolites mainly involved in phenylpropane and flavonoid biosynthesis have been found between two ecotypes of T. hemsleyanum from different regions [16]. In addition, the starch characterizations of T. hemsleyanum tuber roots from different origins showed significant differences on the granule diameter,

Page $2 / 24$ 
amylose content and peak gelatinization temperature, from Zhejiang and Guangxi province [17]. Therefore, authenticity and traceability of geographical origin of $T$. hemsleyanum were of great importance for its quality and medicinal values to prevent mislabeling. The rapid and accurate discrimination of geographical origin of root from T. hemsleyanum would be conducive to the breeding and brand establishment of superior varieties. The growing demand of identifying the provenances of decoction pieces of $T$. hemsleyanum required effective approaches for geographical origin determination, which would significantly contribute to the further development and clinical applications of T. hemsleyanum.

The chloroplast (cp), which performs photosynthesis as well as harbors lots of other metabolic pathways, is an important organelle in plants and is generally non-recombinant and uniparentally inherited [18, 19]. In most angiosperms, chloroplast genome is generally double stranded and circular and the size varies from $120 \mathrm{~kb}$ to $160 \mathrm{~kb}$ [20]. The structure of chloroplast genome is highly conservated, consisting of a small single copy region (SSC), a large single copy region (LSC) and a pair of inverted repeat regions (IR) [21]. The cp genome harbors many different gene loci and non-coding regions containing relatively large amount of DNA sequence information, which has been widely considered as a powerful tool for phylogenetic analysis and further development of species identification and restoration strategies [22]. A great number of cp genomes have been determined and applied in phylogenetic reconstructions from population genetics to investigate the sequence evolution and perform deep divergence analysis at a genera and family level. Comparative chloroplast genome analysis of six Impatiens species reconstructed the taxonomic relationship and provided detailed information about nucleotide diversity hotspots, which could facilitate the systematic evolution research of the Balsaminaceae species [23]. Furthermore, various chloroplast genome regions and DNA barcodes have been considered as useful molecular markers in systematic and population genetic studies to distinguish its closely related species and adulterants [24]. For instance, four chloroplast single nucleotide polymorphism (SNP) variants were identified as powerful markers to differentiate rubber dandelion species from weedy relatives [25]. The DNA barcodes rps16 and trnQUUG were designed based on the comparative analysis of complete chloroplast genome sequences of Conyza bonariensis, which could successfully separate three predominant Conyza species [26]. More importantly, DNA barcodes from chloroplast genome exhibited the potential as a powerful analytical tool for determination of geographical origins. The haplotype map generated by four chloroplast DNA markers identified 10 informative intra specifically variable sites, providing useful molecular tool for tracking Merbau timber originating from peninsular Malaysia [27]. The cp DNA marker trnL-F was successfully applied to investigate phylogeographical pattern of 27 populations of Chrysanthemum indicum across the southwest to northwest areas in China, which revealed fifteen haplotypes and correlative high genetic differentiation among populations [28]. The DNA barcodes from chloroplast genomes would be promising approach to distinguish the geographical origins of $T$. hemsleyanum, as well as rapidly differentiate the genuine and adulterated crude drugs. Therefore, it is highly important and essential to investigate the complete chloroplast genomes of $T$. hemsleyanum from different regions and develop typical molecular markers to discriminate their provenances for its further clinical application and development.

In the current study, we have sequenced and assembled the cp genomes of $T$. hemsleyanum collected from five different regions using Illumina sequencing platform. These sequences were further compared with other known chloroplast genomes from Vitaceae species to reveal the conserved and different features on basic genome structure, codon usage bias, repetitive structure characteristics and IR expansion. The phylogenetic relationship between Tetrastigma species and other closely related taxa from Vitaceae were reconstructed to infer the taxonomic status of Tetrastigma climbers within the families. Finally, we designed two specific DNA barcodes to identify the geographical origins of $T$. hemsleyanum, which successfully divided the T. hemsleyanum climbers from different regions into three clades. The genomic and marker resources described here expanded our understanding of the diversity of chloroplast genomes and their taxonomic relationships within Vitaceae species, and provided an efficient molecular approach for the geographical origin identification of T. hemsleyanum.

\section{Results}

\subsection{Morphological Features and Chloroplast Genome Characteristics of Tetrastigma hemsleyanum}

The samples of Tetrastigma hemsleyanum were collected from Zhejiang, Fujian, Jiangxi, Guangxi and Sichuan provinces, which have been indicated as the main producing zones of $T$. hemsleyanum crude drugs (Fig. 1A). The external morphology and microstructure of the root tubers of $T$. hemsleyanum from different regions were analyzed to identify the differences on morphological features. As shown in Fig. 1B, all of the root tubers from different regions exhibited similar morphological characters, including the root tuber size, the shape of elliptical or spindle, and the epidermis with tan. Moreover, most of root tubers of T. hemsleyanum showed smooth appearance, while a few of them presented folds and lenticel-like protuberances, as well as depressions (Fig. 1B). In addition, the microscopic features of the powder revealed that the cork cells, brown patches, needle crystals of calcium oxalate, starch granules and marginal orifice catheters were abundant in root tubers of T. hemsleyanum from five different regions, while cluster crystals of calcium oxalate were rarely observed in the tubers (Fig. 1C). However, the pharmacognostical analysis failed to identify significant differences on external morphology and microstructure of root tubers of $T$. hemsleyanum from different regions, requiring the development of alternative strategies to discriminate its geographical origin. Therefore, we sequenced the complete cp genome of T. hemsleyanum from five different regions and conducted a comparative analysis to establish and develop potential molecular approach for geographical origins traceability of T. hemsleyanum.

The complete chloroplast sequences of T. hemsleyanum from five different regions of Zhejiang, Fujian, Guangxi, Sichuan and Jiangxi Provinces, have been deposited in the GenBank database with the accession No. of MW375707 MW375711. The size of the whole chloroplast genomes of $T$. 
hemsleyanum varied from 160,124 bp to 160,518 bp, with the smallest and largest T. hemsleyanum cp genome from Jiangxi and Zhejiang Province, respectively. All of the five T. hemsleyanum cp genomes exhibited a typical angiosperm circular chloroplast structure containing four regions: large single-copy region (LSC; 88,131 bp-89,298 bp), small single-copy region (SSC; 18,962 bp-18,965 bp), and a pair of inverted repeats (IR; 26126 bp-26,517 bp) (Fig. 2). A total of 112 genes, including 80 protein-coding genes, 28 tRNAs, and 4 rRNAs were identified from each genome of T. hemsleyanum from different regions (Table 1). The cp genomes showed high similarity in terms of gene contents, orders and orientations. Specifically, the overall GC contents of $T$. hemsleyanum from five regions revealed almost the same results in five regions, among which medicinal plant from Zhejiang, Fujian and Guangxi exhibited a GC content of $37.50 \%$, while that from other two regions showed a result of $37.52 \%$ (Table 2 ). No significant differences on protein coding genes were identified in the $T$. hemsleyanum cp genomes from different regions, with a total length of $80022 \mathrm{bp}$. There were 18 duplicated genes identified in the IR regions of $T$. hemsleyanum cp genome including 8 protein coding genes (rp/2, rp/23, ycf1, ycf2, ycf15, ndhB, rps12 and $r p s 7$ ), 7 tRNA genes (trnA-UGC, trnl-CAU, trnl-GAU, trnL-CAA, trnN-GUU, trnR-ACG and trnV-GAC) and 4 rRNA genes (rrn4.5, rrn5, rrn16, rrn23). (Table 2). Furthermore, 18 distinct genes were indicated as intron-containing genes in the cp genome of $T$. hemsleyanum, including 13 protein coding genes and 5 tRNA genes. All these genes exhibited a single intron, except for $r p s 12, c l p P$ and $y c f 3$ which contained two introns. Moreover, it is intriguing that the location and the intron aera of $r p / 2$ gene were diverse in $T$. hemsleyanum $\mathrm{cp}$ genomes from different genomes. The $r p / 2$ gene of Guanxi and Zhejiang T. hemsleyanum cp genomes possessed two introns and across the junction of IRA and LSC region, which occupied in LSC region with $149 \mathrm{bp}$ and $223 \mathrm{bp}$ respectively. While the $\mathrm{rp} / 2$ gene of $T$. hemsleyanum $\mathrm{cp}$ genome from other three regions showed only one intron and located in IRA completely. The above results indicated the cp genomes of $T$. hemsleyanum from different regions were slightly different, but it is highly conserved on basic structure, genome size, gene number and total GC content.

Table 1

List of genes annotated in the chloroplast genomes of Tetrastigma hemsleyanum

\begin{tabular}{|c|c|c|}
\hline $\begin{array}{l}\text { Classification } \\
\text { of genes }\end{array}$ & Gene Names & Number \\
\hline Photosystem $\otimes$ & psaA, psaB, psaC, psal, psaJ & 5 \\
\hline Photosystem $\otimes$ & $p s b A, p s b B, p s b C, p s b D, p s b E, p s b F, p s b H, p s b l, p s b J, p s b K, p s b L, p s b M, p s b N, p s b T, p s b Z$ & 15 \\
\hline $\begin{array}{l}\text { Cytochrome } \\
\text { b/f complex }\end{array}$ & pet $A$, petB, petD, petG, petL, petN & 6 \\
\hline ATP synthase & atpA, atpB, atpE, atpF, atpH, atpl & 6 \\
\hline $\begin{array}{l}\mathrm{NADH} \\
\text { dehydrogenase }\end{array}$ & $n d h A, n d h B^{*}, n d h C, n d h D, n d h E, n d h F, n d h G, n d h H, n d h l, n d h J, n d h K$ & 11 \\
\hline $\begin{array}{l}\text { RubisCO large } \\
\text { subunit }\end{array}$ & $r b c L$ & 1 \\
\hline $\begin{array}{l}\text { RNA } \\
\text { polymerase }\end{array}$ & rроA, rров, грос1, грос2 & 4 \\
\hline $\begin{array}{l}\text { Ribosomal } \\
\text { proteins (SSU) }\end{array}$ & $r p s 2, r p s 3, r p s 4, r p s 7 *, r p s 8, r p s 11, r p s 12 *, r p s 14, r p s 15, r p s 16, r p s 18, r p s 19$ & 12 \\
\hline $\begin{array}{l}\text { Ribosomal } \\
\text { proteins (LSU) }\end{array}$ & $r p / 2 *, r p / 14, r p / 16, r p / 20, r p / 22, r p / 23 *, r p / 32, r p / 33, r p / 36$ & 9 \\
\hline $\begin{array}{l}\text { Ribosomal } \\
\text { RNAs }\end{array}$ & $r r n 4^{*}, r r n 5^{*}, r r n 16^{*}, r r n 3^{*}$ & 4 \\
\hline $\begin{array}{l}\text { Protein of } \\
\text { unknown } \\
\text { function }\end{array}$ & $y c f 1 *, y c f 2^{*}, y c f 3, y c f 4, y c f 15^{*}$ & 5 \\
\hline Transfer RNAs & 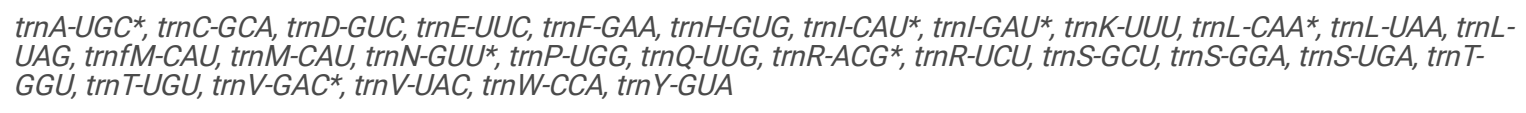 & 28 \\
\hline Other genes & $a c c D, \operatorname{ccs} A, \operatorname{cem} A, \operatorname{clpP}$, infA, matK & 6 \\
\hline Total & & 112 \\
\hline
\end{tabular}


Table 2

Statistics on the basic feature of the cp genomes of five T. hemsleyanum plants and three Vitaceae species

\begin{tabular}{|c|c|c|c|c|c|c|c|c|}
\hline \multirow[t]{2}{*}{ Characteristics } & \multicolumn{5}{|c|}{ Tetrastigma hems/eyanum } & \multirow[t]{2}{*}{ Tetrastigma planicaule } & \multirow{2}{*}{$\begin{array}{l}\text { Ampelopsis } \\
\text { japonica }\end{array}$} & \multirow{2}{*}{$\begin{array}{l}\text { Vitis } \\
\text { vinifera }\end{array}$} \\
\hline & Zhejiang & Fujian & Guangxi & Sichuan & Jiangxi & & & \\
\hline $\begin{array}{l}\text { Genbank } \\
\text { accession No. }\end{array}$ & MW375707 & MW375708 & MW375709 & MW375710 & MW375711 & MW401672 & NC_042235 & NC_007957 \\
\hline $\begin{array}{l}\text { Total length } \\
\text { (bp) }\end{array}$ & 160518 & 160152 & 160153 & 160127 & 160124 & 160323 & 161430 & 160928 \\
\hline $\begin{array}{l}\text { LSC length } \\
\text { (bp) }\end{array}$ & 89298 & 88183 & 88798 & 88131 & 88142 & 88181 & 89626 & 89147 \\
\hline $\begin{array}{l}\text { SSC length } \\
\text { (bp) }\end{array}$ & 18968 & 18965 & 18965 & 18962 & 18962 & 19096 & 18977 & 19065 \\
\hline IR length (bp) & 26126 & 26502 & 26195 & 26517 & 26510 & 26523 & 26413 & 26358 \\
\hline $\begin{array}{l}\text { Gene number } \\
\text { (bp) }\end{array}$ & 112 & 112 & 112 & 112 & 112 & 113 & 114 & 113 \\
\hline $\begin{array}{l}\text { Gene number } \\
\text { in IR regions }\end{array}$ & 19 & 19 & 19 & 19 & 19 & 18 & 19 & 18 \\
\hline $\begin{array}{l}\text { Protein-coding } \\
\text { gene number }\end{array}$ & 80 & 80 & 80 & 80 & 80 & 79 & 80 & 79 \\
\hline $\begin{array}{l}\text { rRNA gene } \\
\text { number }\end{array}$ & 4 & 4 & 4 & 4 & 4 & 4 & 4 & 4 \\
\hline $\begin{array}{l}\text { tRNA gene } \\
\text { number }\end{array}$ & 28 & 28 & 28 & 28 & 28 & 30 & 30 & 30 \\
\hline $\begin{array}{l}\text { Total GC } \\
\text { content (\%) }\end{array}$ & 37.50 & 37.50 & 37.50 & 37.52 & 37.52 & 37.49 & 37.32 & 37.40 \\
\hline
\end{tabular}

To further determine the conserved and variable structures of $\mathrm{cp}$ genome in family Vitaceae, we conducted a comparative analysis between $T$. hemsleyanum plant and other species from tribe Cayratieae (Tetrastigma planicaule), tribe Ampelopsideae (Ampelopsis japonica) and tribe Viteae (Vitis vinifera). The structure of the chloroplast genome appeared to be largely conserved across the family Vitaceae, with little differences on the total genome length, gene number and GC content (Table 2). The size of the chloroplast genome varied from 160,323 bp in T. planicaule to 161,430 bp in A. japonica, and the overall GC content ranged from $37.32 \%$ (A. japonica) to $37.49 \%$ ( $T$. planicaule). However, the types and numbers of genes coded in the cp genomes of $T$. planicaule, $A$. japonica and $V$. vinifera were not identical with that of $T$. hemsleyanum. The lack of ycf 15 gene resulted in a decrease in the number of protein coding genes of $T$. planicaule and $V$. vinifera, while the protein coding gene number of $A$. japonica was consistent with that of $T$. hemsleyanum. In addition, the $y c f 1$ gene of $V$. vinifera completely located in IRB region and was indicated as a pseudogene copy. Compared with the protein coding genes, more significant differences were identified on the the tRNA genes among the cp genomes from the four Vitaceae plants. The trnS-GCU gene was uniquely encoded by $T$. hemsleyanum, while trnG-GCC, trnG-UCC, trnV-GAU were solely encoded by other three Vitaceae species, which led to the uniqueness of T. hemsleyanum.

\subsection{Comparative Analyses of Chloroplast Genome}

As a link between the nucleic acids and proteins, the genetic code plays an important role in the transmission of genetic information in organisms [29] Therefore, we analyzed the codon distribution among the protein coding genes in cp genome of $T$. hemsleyanum from different regions and performed a comparison analysis. The $\mathrm{cp}$ genomes of $T$. hemsleyanum from five regions exhibited almost identical protein-coding sequences, which represented a total of 26,674 codons. All of these codons belonged to 64 codon types and encoded 20 amino acids (Supplementary Fig. 1). However, the numbers of amino acid and the bias of codon usage of T. hemsleyanum cp genomes from different regions exhibited a slight disparity. Leucine was the most abundant amino acid ( $2774 \sim 2776$ codons, $10.40 \% \sim 10.41 \%$ of the total), whereas Cysteine (320 322 codons, $1.20 \% \sim 1.21 \%$ of the total) showed the least abundance in the $\mathrm{cp}$ genome of T. hemsleyanum. Regardless of stop codons, the most commonly applied codon was AUU (1117 1118), encoding isoleucine and the least one was UGC (89 91), encoding cysteine (Supplementary Table 3 ). The single most striking observation to emerge from the data comparison in Supplementary Table 3 was that the codon usage patterns of $T$. hemsleyanum from five different regions could be divided into three types. According to the data in Supplementary Table 3, the codon usage bias of T. hemsleyanum from Jiangxi and Sichuan were exactly the same, while those from Fujian and Guangxi completely displayed the same bias, and the special one from Zhejiang exhibited a unique pattern of codon usage differing from the other regions. Further comparative analysis revealed that a total of 28 variants sites in 21 protein-coding genes of $T$. hemsleyanum from different regions, which led to discrepancies in codon usage preference and the number of amino acid coding (Supplementary Table 4). What stands out in the Supplementary Table 4 was that, the protein coding genes of atpB, ccsA, ycf2 and $y c f 1$ exhibited two variable sites while $a c c D$ gene displayed 3 mutation sites. The more surprising correlation was the variant sites of accD gene in $T$. hemsleyanum from Jiangxi and Sichuan provinces resulted in the encoding of lysine, which was obviously distinguished with methionine, glutarnine and asparagine encoded in cp genome from other three regions. Comparing with T. hemsleyanum of Sichuan, Jiangxi and Zhejiang regions, one base variation was 
identified in the $n d h D$ and $y c f 2$ genes of that from Fujian and Guangxi regions, which led to the preference of GGG and GGC, respectively (Supplementary Table 4). Taken together, these results provided important insights into the understanding of protein adaptive evolution and strategy development of identification geographical origin of T. hemsleyanum.

Previous reports have indicated that codon usage bias of chloroplast genome may be affected by selection, mutation and random drift [30, 31]. Further comparing analysis between $T$. hemsleyanum and other three Vitaceae species suggested the coded amino acids of $T$. planicaule, $A$. japonica and $V$. vinifera were identical with that of $T$. hemsleyanum. The numbers of codons in the cp genome of T. planicaule, A. japonica and $V$. vinifera were 26978 , 26990 and 26124, respectively (Supplementary Table 3). On average, the most abundant amino acids in the three species were leucine (T. planicaule 2800, 10.38\%; A. japonica 2724, 10.09\%; V. vinifera 2803, 10.73\%) whereas the least abundant amino acid was Cysteine (T. planicaule $327,1.21 \%$; $A$. japonica $308,1.14 \%$; V. vinifera 325, 1.24\%). In addition, similar codon usage patterns were observed among the eight Vitaceae plants. As shown in Supplementary Table 3, most of the amino acid showed codon preferences in the cp genomes of Vitaceae plants. However, methionine (AUG) and tryptophan (UGG) were encoded by only one codon and exhibited no codon preferences. AGA (1.87 1.90) in arginine showed the highest RSCU value, and the lowest one was AGC $(0.34 \sim 0.36)$ in serine. Moreover, the RSCU value for each Vitaceae species exhibited similar codon preference in the 64 codons in the CDS genes. As a result, 31 of them for each species exhibited greater preference (RSCU $>1$ ), indicating an obvious codon bias in the amino acids. Most (29 codons) of these preferred codons among eight Vitaceae plants species ended with the nucleotide of $\mathrm{A}$ or $\mathrm{U}$. Therefore, the investigation on codon preferences is conducive to understand the exogenous gene expression and the molecular evolution mechanisms of $T$. hemsleyanum in Vitaceae.

Contraction and expansion of the IR region is a common phenomenon known as ebb and flow, which could be used as effective tool for phylogenetic relationship and classification research of medicinal plants [32]. A comparison of five T. hemsleyanum plants and three Vitaceae species for borders was performed between the IRs and two single copies regions in detail. The length of the IR regions was similar among the eight Vitaceae species ranging from $26,126 \mathrm{bp}$ in $\mathrm{T}$. hemsleyanum (Zhejiang) to $26,523 \mathrm{bp}$ in T. planicaule, with certain expansion and contraction (Fig. 3). Particularly, some notable differences were found at the boundaries among $\mathrm{cp}$ genomes of $T$. hemsleyanum from different regions. The LSC-IRb border was located within the rps19 gene in T. hemsleyanum from Jiangxi, Fujian and Sichuan province, with extending length of 96 bp, 92 bp and 103 bp to the IRB region, respectively (Fig. 3). However, the rps19 gene was completely encoded in LSC region and exhibited 290 and 216 bp distance to the junction of the LSC-IRB region in the cp genome of T. hemsleyanum from Zhejiang and Guangxi, respectively (Fig. 3). In contrast, the locating position of ycf1 gene was highly conserved at the boundary of IR/SSC region among Vitaceae plants except that of V. vinifera, which exhibited a pseudogene gene with the size of 1030 bp completely locating in IRB region (Fig. 3). The contraction and expansion of ycf1 gene at the boundary of LSC-IRB within five $T$. hemsleyanum plants were identical, which occupied 1,140 bp and 29 bp in IRB and SSC regions, respectively. Another interesting observation is that the overlap between $y c f 1$ gene and the IRA region was significantly longer of other three species than that of $T$. hemsleyanum, which showed 1,144 bp for $T$. planicaule and 1,116 bp for $A$. japonica and $V$. vinifera, respectively. However, the overlap length was only 34 bp in the $c p$ genome of $T$. hemsleyanum, which could be considered to be one of the reasons for the length change among these Vitaceae cp genomes.

\subsection{Repeat Sequences Analysis and RNA editing Sites identification}

Long repeats are significant genetic resources, playing a crucial role in genome rearrangement and intermolecular recombination [33]. As shown in Supplementary Fig. 2, the long repeat sequences detected in T. hemsleyanum cp genomes of Jiangxi and Sichuan revealed the identical results, while specimens from Zhejiang, Fuajin and Guangxi regions exhibited slightly different types and number of repeat sequences. Within the five T. hemsleyanum plants, the long repeats analysis revealed the most abundant repeats were length of 30-39, with the largest number in type of forward repeats (27-28), followed by palindrome (18-19), complemented (2) and reverse (2) repeats. These results further confirmed the high similarities on the type of repeats and certain slight variations on terms of the number and length among cp genomes of T. hemsleyanum from different regions (Supplementary Fig. 2A). However, the comparison of T. hemsleyanum with the other three Vitaceae species displayed an obvious distinction. A total of 49, 48, 40 long repeats were identified in the cp genomes of $T$. planicaule, A. japonica and $V$. vinifera respectively. In contrast with the cp genome of T. hemsleyanum, no complemented repeats were determined in the $\mathrm{cp}$ genome of other three Vitaceae plants. In addition, the type of reverse repeats was also lost in the cp genome of T. planicaule. Among these Vitaceae plants, most of the repeat units were short, ranging from 30 to 59 bp (Supplementary Fig. 2B).

Simple sequence repeats (SSRs) play an essential role in plant taxonomy and population genetics studies for the high polymorphism and codominance [34]. In total, 56 SSRs were identified in the cp genomes of T. hemsleyanum plants from four regions, while the species from Guangxi exhibited a SSR number of 57. The majority of SSR sequences were mononucleotide repeats (42-43), followed by dinucleotides (11) and tetranucleotides (3) (Table 3). The cp genome of T. hemsleyanum of Jiangxi and Sichuan exhibited the identical results on SSR types and numbers. However, the distinctions of SSRs in T. hemsleyanum cp genomes from the other regions were embodied in SSRs count of mononucleotide repeats (Fig. 4A). Specifically, the numbers of A/T repeats in the cp genomes of T. hemsleyanum plants from Jiangxi, Zhejiang, Fujian, Guangxi and Sichuan were 42, 41, 41, 42 and 42, respectively. In addition, the samples from Jiangxi and Sichuan showed no C/G SSR repeats in the cp genomes. These results further indicated that SSR might be a useful molecular marker for species determination of geographical origins of $T$. hemsleyanum. In addition, a comparative SSRs analysis conducted with three Vitaceae species revealed 55, 69 and 54 SSRs were detected in the cp genomes of $T$. planicaule, $A$. japonica and $V$. vinifera, respectively (Table 3). It is must mentioned that $T$. planicaule from Tetrastigma genus showed identical SSRs types with slight distinctions on SSR quantities (Fig. 4B). Comparing with the Tetrastigma plants, $A$. japonica and $V$. vinifera possessed lots of additional types of SSRs and repeat nuits, containing mono-(45/35), di-(13/8), tri-(4/5), tetra-(4/5) and penta-(3/1) respectively. The extra SSR

Page $6 / 24$ 
sequences include unique AAT/ATT, AGC/CTG, AAG/CTT, AATC/ATTG, AGAT/ATCT, AAAAT/ATTTT and AATAT/ATATT in A. japonica cp genome and AAT/ATT, AGC/CTG, AATC/ATTG, ACAT/ATGT, AGAT/ATCT and AGGAT/ATCCT in V. vinifera cp genome, respectively (Fig. 4B). Moreover, the lack of $\mathrm{AG} / \mathrm{CT}$ and AATT/AATT in both of $A$. japonica and $V$. vinifera also revealed the discrepancy of SSR loci among different genus. Among all Vitaceae species, the number of SSRs composed by A/T were significantly greater than that containing $\mathrm{G}$ or $\mathrm{C}$, indicating that the base composition of SSRs was biased toward the application of A/T bases, which was consistent with A-T enrichment in complete chloroplast genomes [35].Taken together, these results provided important insights into understanding intrageneric and intergeneric variations within $T$. hemsleyanum and its relatives in Vitaceae species.

Table 3

The number and types of SSR in five T. hemsleyanum plants and three Vitaceae species

\begin{tabular}{|c|c|c|c|c|c|c|c|c|c|}
\hline \multirow{2}{*}{$\begin{array}{l}\text { SSR } \\
\text { type }\end{array}$} & \multirow[t]{2}{*}{ Repeat unit } & \multicolumn{3}{|c|}{ Amount } & \multirow[b]{2}{*}{$\begin{array}{l}\text { T.h. } \\
\text { (Guangxi) }\end{array}$} & \multirow[b]{2}{*}{$\begin{array}{l}\text { T.h. } \\
\text { (Sichuan) }\end{array}$} & \multirow[b]{2}{*}{$\begin{array}{l}\text { T. } \\
\text { planicaule }\end{array}$} & \multirow[b]{2}{*}{$\begin{array}{l}\text { A. } \\
\text { japonica }\end{array}$} & \multirow[b]{2}{*}{$\begin{array}{l}V \\
\text { vinifera }\end{array}$} \\
\hline & & $\begin{array}{l}\text { T.h. } \\
\text { (Jiangxi) }\end{array}$ & $\begin{array}{l}\text { Th.h. } \\
\text { (Zhejiang) }\end{array}$ & $\begin{array}{l}\text { Th. } \\
\text { (Fujian) }\end{array}$ & & & & & \\
\hline \multirow[t]{2}{*}{ Mono } & $\mathrm{A} / \mathrm{T}$ & 42 & 41 & 41 & 42 & 42 & 39 & 45 & 34 \\
\hline & $\mathrm{C} / \mathrm{G}$ & I & 1 & 1 & 1 & I & I & / & 1 \\
\hline \multirow[t]{2}{*}{$\mathrm{Di}$} & $\mathrm{AG} / \mathrm{CT}$ & 1 & 1 & 1 & 1 & 1 & 1 & / & / \\
\hline & AT/AT & 10 & 10 & 10 & 10 & 10 & 11 & 13 & 8 \\
\hline \multirow[t]{3}{*}{ Tri } & AAT/ATT & l & l & l & l & / & / & 2 & 4 \\
\hline & AGC/CTG & I & / & / & / & / & / & 1 & 1 \\
\hline & AAG/CTT & / & / & / & I & / & / & 1 & / \\
\hline \multirow[t]{5}{*}{ Tetra } & AAAT/ATTT & 2 & 2 & 2 & 2 & 2 & 2 & 2 & 1 \\
\hline & AATC/ATTG & I & / & I & / & / & / & 1 & 1 \\
\hline & AATT/AATT & 1 & 1 & 1 & 1 & 1 & 2 & / & / \\
\hline & ACAT/ATGT & I & / & l & l & I & l & / & 1 \\
\hline & AGAT/ATCT & I & I & / & / & / & / & 1 & 2 \\
\hline \multirow[t]{3}{*}{ Penta } & AGGAT/ATCCT & I & / & I & I & / & I & / & 1 \\
\hline & AAAAT/ATTTT & I & I & l & l & / & I & 1 & / \\
\hline & AATAT/ATATT & / & / & / & / & / & I & 2 & I \\
\hline Total & & 56 & 56 & 56 & 57 & 56 & 55 & 69 & 54 \\
\hline
\end{tabular}

The RNA editing process is an essential maturation mechanism to avoid incorrect RNA mutations and is widespread in the chloroplast genome of plants [36]. In total, 71 potential RNA editing sites have been predicted in 24 protein-coding genes of the cp genome of T. hemsleyanum, which displayed no distinction in numbers of RNA editing sites and conversions of amino acids in $\mathrm{cp}$ genome of $T$. hemsleyanum from different regions. (Table 4). Among the 71 RNA editing sites, 17 codons were observed to be edited at the first nucleotide position, whereas 54 codons were identified to be edited at the second nucleotide position, and no codons were edited at both of the first and second nucleotide. All of the identified codon changes in the cp genomes of $T$. hemsleyanum showed $\mathrm{C}$ to $\mathrm{T}$ conversions. Especially, the $n d h B$ gene showed the largest number of RNA editing sites (11 editing sites), followed by $n d h D$ (8 editing sites) and $n d h F$ (7 editing sites), while nine genes (accD, atpl, atpF, $c c s A, c l p P, p s b E, p s b F, p s b L$ and $r p / 20$ ) exhibited only one editing site in $T$. hemsleyanum (Table 4). The RNA edition on protein gene resulted in a total of 11 kinds of amino acid conversions in the cp genome of $T$. hemsleyanum. The conversions of $\mathrm{H}$ to $\mathrm{Y}, \mathrm{L}$ to $\mathrm{F}, \mathrm{P}$ to $\mathrm{S}, \mathrm{R}$ to $\mathrm{W}, \mathrm{R}$ to $\mathrm{C}$ were due to codons edited at the first nucleotide position, while the $\mathrm{S}$ to $\mathrm{L}, \mathrm{P}$ to $\mathrm{L}, \mathrm{S}$ to $\mathrm{F}, \mathrm{T}$ to $\mathrm{M}, \mathrm{A}$ to $\mathrm{V}, \mathrm{T}$ to I conversions were caused by codons edited at the second nucleotide position (Supplementary Table 5 ). The conversion of serine to leucine ( $\mathrm{S}$ to $\mathrm{L}$ ) was the most abundant kind of conversion, accounting for $42.3 \%$, while arginine to tryptophan ( $\mathrm{R}$ to $\mathrm{W}$ ) and arginine to cysteine ( $\mathrm{R}$ to $\mathrm{C}$ ) were the least conversion, accounting for $1.4 \%$ merely (Supplementary Table 5 ). Furthermore, the predicted RNA editing sites in the cp genomes of $T$. planicaule, $A$. japonica and $V$. vinifera showed similar results with that of $T$. hemsleyanum, with the RNA editing sites number of 71,72 and 70 respectively. The slight difference of the number of RNA editing sites were observed in $a c c D, n d h B$ and $n d h F$ genes among these Vitaceae plants, which led to the distinctions of amino acid conversions (Supplementary Table 5). Since the close correlation between RNA editing sites and nucleotide substitution of protein coding genes, we performed further analysis to investigate the synonymous substitutions (Ks) and non-synonymous substitutions (Ka) of protein coding genes with abundant RNA editing sites. The Ka/Ks ratios of most genes (22/24) in $T$. hemsleyanum were less than 0.5 expect the matK (0.5534) and rps16 (0.5687), suggesting an obvious purifying selection pattern. Particularly, the $c l p P, p s b L$ and $p s b F$ genes even exhibited a $\mathrm{Ka} / \mathrm{Ks}$ value of 0 , showing the three genes were possibly under strong purifying selection pressure (Table 5). 
Table 4

Number of the RNA editing sites in the cp genome of $T$. hemsleyanum and three Vitaceae species

\begin{tabular}{|c|c|c|c|c|}
\hline \multirow[t]{2}{*}{ Gene } & \multicolumn{4}{|c|}{ Number of RNA editing sites } \\
\hline & T. hemsleyanum & T.planicaule & A. japonica & V. vinifera \\
\hline$a c c D$ & 1 & 1 & 2 & 1 \\
\hline $\operatorname{atp} A$ & 3 & 3 & 3 & 3 \\
\hline $\operatorname{atp} F$ & 1 & 1 & 1 & 1 \\
\hline atpl & 1 & 1 & 1 & 1 \\
\hline $\operatorname{ccs} A$ & 1 & 1 & 1 & 1 \\
\hline$c l p P$ & 1 & 1 & 1 & 1 \\
\hline matk & 4 & 4 & 4 & 4 \\
\hline$n d h A$ & 4 & 4 & 4 & 4 \\
\hline$n d h B$ & 11 & 11 & 12 & 11 \\
\hline$n d h D$ & 8 & 8 & 8 & 8 \\
\hline$n d h F$ & 7 & 7 & 6 & 6 \\
\hline$n d h G$ & 3 & 3 & 3 & 3 \\
\hline petB & 2 & 2 & 2 & 2 \\
\hline$p s b E$ & 1 & 1 & 1 & 1 \\
\hline$p s b F$ & 1 & 1 & 1 & 1 \\
\hline$p s b L$ & 1 & 1 & 1 & 1 \\
\hline rp/20 & 1 & 1 & 1 & 1 \\
\hline rроA & 2 & 2 & 2 & 2 \\
\hline$r p o B$ & 5 & 5 & 5 & 5 \\
\hline rpoC1 & 2 & 2 & 2 & 2 \\
\hline rpoc2 & 4 & 4 & 4 & 4 \\
\hline rps2 & 2 & 2 & 2 & 2 \\
\hline rps14 & 2 & 2 & 2 & 2 \\
\hline rps16 & 3 & 3 & 3 & 3 \\
\hline Total & 71 & 71 & 72 & 70 \\
\hline
\end{tabular}


Table 5

The value of $\mathrm{Ka} / \mathrm{Ks}$ in 25 protein coding genes with RNA editing sites in T. hemsleyanum (Jiangxi)

\begin{tabular}{|c|c|c|c|c|}
\hline Gene & $\begin{array}{l}\text { Number of } \\
\text { RNA editing sites }\end{array}$ & non-synonymous substitutions (Ka) & synonymous substitutions (Ks) & $\mathrm{Ka} / \mathrm{Ks}$ \\
\hline$n d h B$ & 11 & 0.0113 & 0.0243 & 0.4650 \\
\hline$n d h D$ & 8 & 0.0516 & 0.3257 & 0.1584 \\
\hline$n d h F$ & 7 & 0.1087 & 0.3022 & 0.3597 \\
\hline$r p o B$ & 5 & 0.0240 & 0.2243 & 0.1070 \\
\hline$n d h A$ & 4 & 0.0383 & 0.2928 & 0.1308 \\
\hline matK & 4 & 0.1296 & 0.2342 & 0.5534 \\
\hline rpoc2 & 4 & 0.0704 & 0.2663 & 0.2644 \\
\hline$n d h G$ & 3 & 0.0491 & 0.2718 & 0.1807 \\
\hline atpA & 3 & 0.0334 & 0.2751 & 0.1214 \\
\hline rps16 & 3 & 0.1109 & 0.1950 & 0.5687 \\
\hline гроA & 2 & 0.0700 & 0.1879 & 0.3725 \\
\hline rpoC1 & 2 & 0.0267 & 0.2567 & 0.1040 \\
\hline petB & 2 & 0.0124 & 0.1742 & 0.0712 \\
\hline rps2 & 2 & 0.0111 & 0.2074 & 0.0535 \\
\hline rps14 & 2 & 0.0263 & 0.1387 & 0.1896 \\
\hline accd & 1 & 0.1093 & 0.2714 & 0.4027 \\
\hline atpF & 1 & 0.0469 & 0.1480 & 0.3169 \\
\hline atpl & 1 & 0.0263 & 0.1612 & 0.1631 \\
\hline $\operatorname{ccs} A$ & 1 & 0.0844 & 0.2810 & 0.3004 \\
\hline$c l p P$ & 1 & 0 & 0 & 0 \\
\hline$p s b E$ & 1 & 0.0053 & 0.2050 & 0.0259 \\
\hline$p s b F$ & 1 & 0 & 0.0934 & 0 \\
\hline$p s b L$ & 1 & 0 & 0 & 0 \\
\hline rp/20 & 1 & 0.0709 & 0.1643 & 0.4315 \\
\hline
\end{tabular}

\subsection{Phylogenetic Analysis}

The previous reports by molecular and morphological data indicated the family of Vitaceae could be classified into five major clades, including the tribe of Ampelopsideae, Cisseae, Cayratieae, Parthenocisseae, and Viteae [37]. However, the deep phylogenetic relationship of Vitaceae still needs further explorations to reveal the evolutionary characters and genetic status of grape species. Therefore, we constructed phylogenetic tree of Viteae family based on the 70 protein-coding gene datasets by maximum likelihood (ML) and maximum parsimony (MP) method, respectively. These grape plants contained 4 species from tribe Viteae, 3 species from tribe Ampelopsideae and 6 plants from tribe Cayratieae. Melaleuca alternifolia and $M$. cajuputi were chosen as the outgroups for phylogenetic analysis. As shown in Fig. 5, nearly all of the nodes received moderate to high support values in the ML and MP tree analysis. However, several topological differences have been occurred in relationships within the five T. hemsleyanum species and the tribe of Viteae between the ML and MP tree results (Fig. 5). The phylogenetic analysis among the five T. hemsleyanum plants revealed a stable monophyletic group with high bootstrap values, which exhibited a stable sister relationship with T. planicaule, indicating a close genetic relationship within the genus of Tetrastigma (Fig. 5). In addition, the ML analysis indicated that the samples of T. hemsleyanum from Fujian and Sichuan regions clustered together to form a combined group with a bootstrap score of 68 , which subsequently gathered together with $T$. hemsleyanum species from other three regions (Fig. 5A). These results indicated certain subtle protein coding differences of $T$. hemsleyanum cp genomes from different regions, providing potential molecular tools for distinguishing the geographical origins of T. hemsleyanum. Furthermore, the Ampelopsideae species and Viteae plants combined together to form a clade with strong statistical support, which combined with six Tetrastigma plants to form a robust monophyletic clade, which was consist with the previous classification of the tribes of Ampelopsideae, Viteae and Cayratieae in Vitaceae.

\subsection{Nucleotide Diversity Analysis and Development of Molecular Marker for Geographical Origin Discrimination}


The complete $\mathrm{cp}$ genomes with high variable levels provides potential molecular marker for species identification and geographical origin determination. In order to assess the sequences divergence level within the Vitaceae species, the complete cp genomes have been multiple aligned and applied DnaSP to calculate nucleotide variability (Pi). As shown in Fig. 6A, the sliding window analysis revealed 5 highly variable regions with $\mathrm{Pi}$ values ranging from 0.06194 to 0.10611 across four complete cp genomes of Vitaceae species, including 4 intergenic regions ( $r p s 16$-trnQ, psbM-trnD, psbZtrnfM and ycf3-trnS) and one protein coding genes (ycf1) (Fig. 6A). Among the five mutational hotspot loci, four highly variable hotspots were located in the LSC region, and ycf1 gene with the Pi value of 0.06194 was identified in the SSC region. However, none of the hypervariable loci were determined in the IR region, further confirmed that the IR regions were highly conserved in the cp genomes among the Vitaceae species. The $r p s 16$-trnQ gene exhibited the highest Pi value of 0.10611 , followed by psbZ-trnfM and ycf3-trnS with the Pi values of 0.10083 and 0.10056 , respectively. Besides, a comparative analysis was carried out to determine the numbers of SNP sites and Gaps to further explore the characteristics of five hypervariable regions among four Vitaceae plants. The five mutational hotspots in the cp genome of $T$. hemsleyanum from Zhejiang province ranged from 892 bp (psbZ-trnfM) to $1139 \mathrm{bp}$ (rps16-trnQ) in length (Table 6). Apparently, the high variable sequences of T. planicaule from Tetrastigma genus exhibited a small number of SNP sites (3-8) and Gaps (0-19) than that of T. hemsleyanum from Jiangxi Province except $p s b M$-trnD region, which contained the SNP site and Gaps of 79 and 39 in the cp genome of $T$. planicaule, respectively. However, a great deal of variable sites was displayed in the 5 mutational hotspots of $A$. japonica and $V$. vinifera. For instance, the hypervariable regions of psbZ-trnfM showed 104 and 115 SNP sites in $A$. japonica and $V$. vinifera, respectively, which was significantly higher than that of $T$. hemsleyanum of Zhejiang. All these discrepancies led to variable mutational hotspot lengths in the Vitaceae plants eventually, and also provided potential molecular markers to resolve the difficulties in species identification of Vitaceae species.

Table 6

Multiple analysis of the mutational hotspots in four Vitaceae plants

\begin{tabular}{|c|c|c|c|c|c|}
\hline mutational hotspots & Specises & Length & $\begin{array}{l}\text { GC } \\
\text { content }\end{array}$ & Number of SNP sites & $\begin{array}{l}\text { Total length of } \\
\text { Gaps }\end{array}$ \\
\hline \multirow[t]{4}{*}{ rps16-trnQ } & T. hemsleyanum (Jiangxi) & 1139 bp & $23.09 \%$ & / & / \\
\hline & T. planicaule & 1141 bp & $23.14 \%$ & 5 & 4 \\
\hline & A. japonica & 1208 bp & $20.86 \%$ & 149 & 167 \\
\hline & V. vinifera & 1076 bp & $21.84 \%$ & 98 & 138 \\
\hline \multirow[t]{4}{*}{ psbM-trnD } & T. hemsleyanum (Jiangxi) & 895 bp & $35.08 \%$ & / & / \\
\hline & T. planicaule & 868 bp & $35.37 \%$ & 79 & 39 \\
\hline & A. japonica & 860 bp & $33.37 \%$ & 60 & 77 \\
\hline & V. vinifera & 843 bp & $34.28 \%$ & 41 & 100 \\
\hline \multirow[t]{4}{*}{ psbZ-trnfM } & T. hemsleyanum (Jiangxi) & 892 bp & $24.33 \%$ & / & / \\
\hline & T. planicaule & 883 bp & $24.46 \%$ & 8 & 19 \\
\hline & A. japonica & 907 bp & $24.33 \%$ & 104 & 95 \\
\hline & V. vinifera & 911 bp & $22.39 \%$ & 115 & 69 \\
\hline \multirow[t]{4}{*}{ ycf3-trns } & T. hemsleyanum (Jiangxi) & 1031 bp & $33.56 \%$ & / & / \\
\hline & T. planicaule & 1029 bp & $33.92 \%$ & 3 & 2 \\
\hline & A. japonica & 1113 bp & $33.33 \%$ & 97 & 128 \\
\hline & V. vinifera & 1123 bp & $33.93 \%$ & 97 & 146 \\
\hline \multirow[t]{4}{*}{ ycf1 } & T. hemsleyanum (Jiangxi) & 977 bp & $30.60 \%$ & / & / \\
\hline & T. planicaule & 977 bp & $30.40 \%$ & 6 & 0 \\
\hline & A. japonica & 965 bp & $29.95 \%$ & 77 & 12 \\
\hline & V. vinifera & 980 bp & $30.61 \%$ & 67 & 15 \\
\hline
\end{tabular}

To determine the potential of variable sequences in $\mathrm{cp}$ genome for geographical origin discrimination, we further evaluate the the sequences divergence level of $T$. hemsleyanum from different regions. The results demonstrated that the intraspecific differences of $T$. hemsleyanum was much lower than interspecific differences among Vitaceae species (Fig. 6). A total of 5 mutational hotspots with relative high Pi value ( $\geq 0.004$ ) have been screened out in T. hemsleyanum plants, including 2 hypervariable regions (trnL-CAA and trnN-GUU) located in IRs and one intergenic region located in SSC (ndhD-psaC) with the Pi value of 0.009 (Fig. 6B). Moreover, we found that both the SSC and IR regions were more variable than the LSC region in the chloroplast genomes of $T$. hemsleyanum from different regions. This result was significant different with the general observations in other species, 
where the IR regions usually exhibited lower variability than the LSC and SSC regions. Accordingly, these hypervariable regions with abundant intraspecific variable sites could be developed as potential DNA barcodes to discriminate the geographical origins of T. hemsleyanum.

Our study designed five DNA barcodes ( $a c c D, \operatorname{trn} L, \operatorname{trnN}, n d h D-p s a C$ and $n d h C$-trnV) based on hypervariable regions for PCR amplification of $T$. hemsleyanum medicinal materials in the Zhejiang region (Fig. 7A). The single bright band in agarose gel electrophoresis implied amplification of accD, $t r n L$ and $t r n N$, while the $t r n L$ and $t r n N$ showed higher PCR amplification efficiency and sequence diversity. As a result, the two DNA barcodes were amplified with DNA of $T$. hemsleyanum samples from six different regions in batches to further analyze the efficiency of geographical origin discrimination. The detailed sequence information of the two PCR products is shown in Table 7. The size of the $\operatorname{trn} L$ and $\operatorname{trnN} \mathrm{Nbarcodes}$ in all $T$. hemsleyanum samples was $1143 \mathrm{bp}$ and $469 \mathrm{bp}$, respectively. The trnL sequences of $T$. hemsleyanum from Sichuan exhibited a unique GC content of $38.76 \%$, while remaining $T$. hemsleyanum samples from other regions showed a GC content of $38.85 \%$ in trnL (Table 7 ). A total of five stable variants at position of $165 \mathrm{bp}, 166 \mathrm{bp}, 167 \mathrm{bp}, 168 \mathrm{bp}$ and $1036 \mathrm{bp}$ were identified in the trnL sequence, generating three haplotypes of T. hemsleyanum from different regions (including our experiments and data from GenBank). The trnL sequences of T. hemsleyanum from Sichuan Province exhibited a unique haplotype of A3, while those from Zhejiang Province displayed two haplotypes of A1 and A2 (Table 7). Notably, that T. hemsleyanum plants from Jiangxi, Zhejiang, Fujian, Guangxi and Guangdong regions harbored the identical trnL haplotype of $A 1$, indicating the genetic variation of $A 1$ was the main variety distributed in China due to its strong environmental adaptability. Additionally, the trnN sequences of $T$. hemsleyanum from different origins showed an identical GC content of $43.50 \%$. However, these $t r n N$ sequences exhibited four variable bases at the position of 164 bp, 165 bp, 166 bp and $167 \mathrm{bp}$, generating 2 haplotypes among different regions (Supplementary Table 6). Interestingly, the trnN sequence from Sichuan region showed a unique haplotype of B2, while that from other regions exhibited the same haplotype of B1 (Table 7). These results demonstrated that the intraspecies discrepancy of $T$. hemsleyanum plants among different regions, further confirming the availability and necessity of geographical origin identification strategy based on molecular markers of chloroplast genome. 
Table 7

Sequence analysis of T. hemsleyanum samples and other Vitaceae species basing on two DNA barcodes

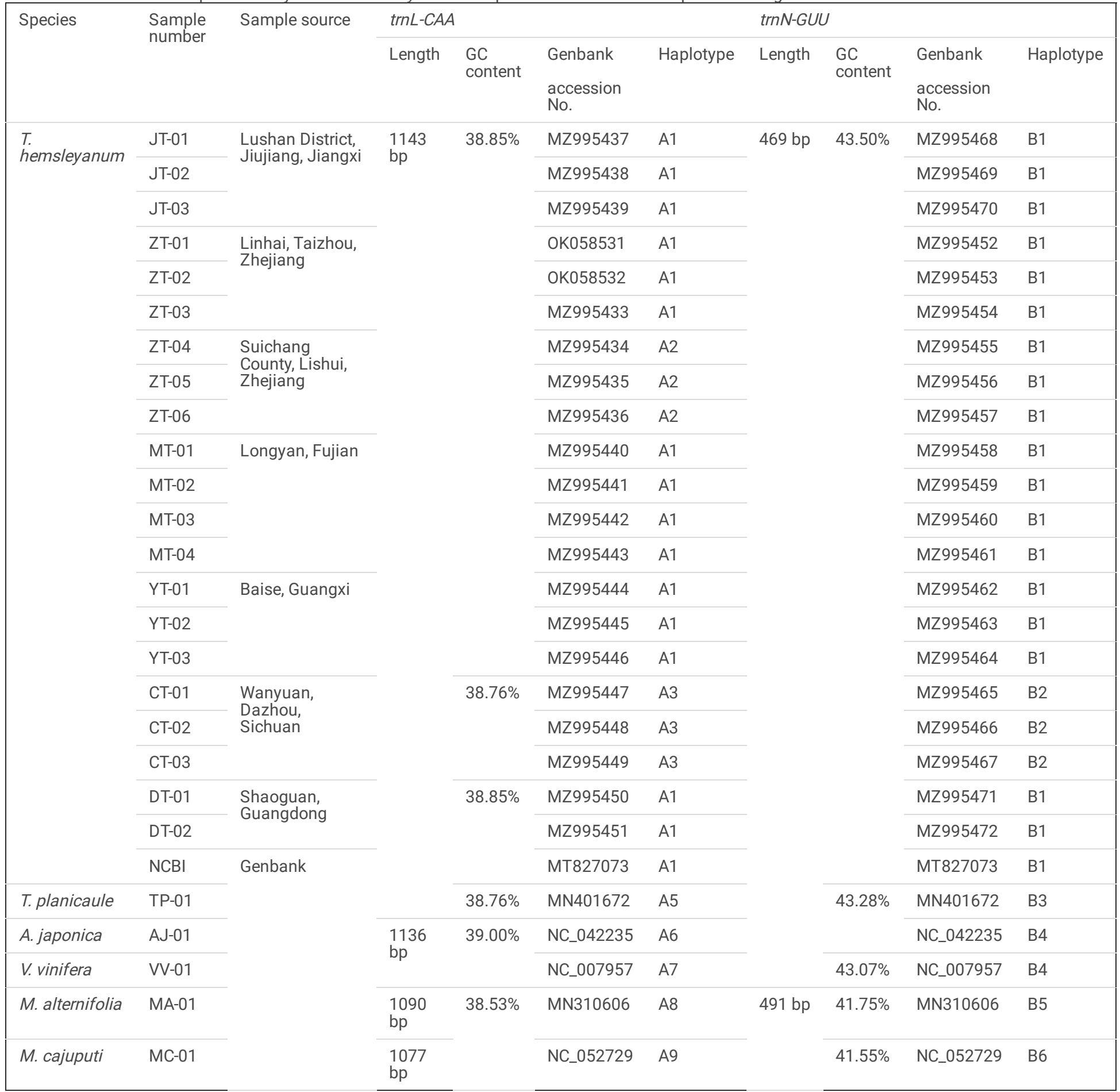

This study also explored the genetic distance of intraspecific and interspecific variation within the $\operatorname{trn} L$ and $\operatorname{trnN}$ sequences of the $T$. hemsleyanum medicinal materials from different regions (Figs. 7B, C, D). The K2P distance of both $t r n L$ and $t r n N$ sequences among the $21 \mathrm{~T}$. hemsleyanum samples ranged from 0.000 to 0.004 , suggesting a significant barcoding gap among plants from different regions. For instance, the divergence value of trn $L$ was highest (0.004) between the Jiangxi and Zhejiang regions and lowest (0.001) between the Jiangxi and Sichuan regions. The intraspecific genetic distance of $\operatorname{trnN}$ sequence between Jiangxi and the Sichuan region had a K2P value of 0.004, suggesting a barcoding gap. However, both of the two cp molecular markers failed to generate barcoding gap among species from Jiangxi, Fujian, Guangxi and Guandong province, indicating the inability of discriminating geographical origin from these regions by $\operatorname{trn} L$ and $t r n N$. Moreover, the combination of $\operatorname{trn} L$ and $\operatorname{trn} N$ sequences revealed a lower intraspecific distance among different geographical origin of $T$. hemsleyanum than the single molecular marker (Fig. 7D). The intraspecific genetic distances based on $t r n L+t r n N$ sequences between Jiangxi and Zhejiang, Jiangxi and Sichuan, and among Jiangxi, Fujian, Guangxi, Guangdong and Zhejiang were $0.002,0.003$ and 0 , respectively (Fig. 7D). The interspecific distance was greater among the Vitaceae species than that intraspecific distance of $T$. hemsleyanum samples, suggesting the developed DNA barcodes could be successfully applied for $T$. hemsleyanum species identification from other Vitaceae plants (Fig. 7D). The NJ tree analysis of the $t r n L$ and $\operatorname{trn} N$ barcodes revealed a clear distinction clearly among the 
different geographical origins of T. hemsleyanum plants (Figs. 7E, F). The trnL-based NJ tree generated three groups with different geographical origins, while $t r n N$-based NJ tree only provided two clades of $T$. hemsleyanum plants. The Clade I of trnL-based NJ tree consisted of all $T$.

hemsleyanum samples from Jiangxi, Fujian, Guangxi and Guangdong areas, three samples from Zhejiang (ZT-01, ZT-02 and ZT-03) and one sample from Genbank (NCBI) with the bootstrap support value of 60. Clade II included T. hemsleyanum samples from Sichuan and T. planicaule samples. The other three T. hemsleyanum samples from Zhejiang (ZT-04, ZT-05 and ZT-06) formed Clade III (Fig. 7E). The Clade I of the trnN-based NJ tree included all samples from Sichuan, while Clade II consisted of the samples from the other five regions and the sample (NCBI) from the Genbank (bootstrap score, 93). Although trnL barcode is more powerful in discriminating geographical origins of $T$. hemsleyanum than the trnN barcode, it failed to distinguish other Vitaceae species from T. hemsleyanum (Fig. 7E). Finally, we constructed the NJ tree based on the combination sequence of trnL + $\operatorname{trnN}$ to determine the identification accuracy for T. hemsleyanum plants from different regions. Interestingly, this NJ tree divided the T. hemsleyanum plants into three groups. The three samples from Zhejiang province (ZT-04 to ZT-06) and Sichuan samples (CT-01 to CT-03) each formed a separate group, while the other samples from Zhejiang province (ZT-01 to ZT-03) and samples from other regions formed the third group (Fig. 8). Furthermore, the position of each Vitaceae species in the NJ tree based on a combination barcode was similar to phylogenetic trees based on the 70 protein-coding gene datasets (Figs. 5, 8). The NJ tree clearly showed that the Tetrastigma genus (T. planicaule and T. hemsleyanum samples) formed the main branch (bootstrap score, 54). They were significantly distinguished from the representative species of the Viteae tribe and Ampelopsideae tribe. This is an indication of the species identification potential of the combined molecular markers. These results demonstrate that the combined barcode strategy of $t r n L+t r n N$ derived from comparative chloroplast genomes is a potential molecular tool for the geographical origin discrimination of $T$. hemsleyanum in China.

\section{Discussion}

\subsection{Chloroplast Genome Features of Tetrastigma and Vitaceae Plants}

The chloroplast genomes of the five T. hemsleyanum plants with different geographical origins as well as other representative species of Tetrastigma and Vitaceae exhibited a typical quadripartite structure containing a pair of IR regions, a single LSC region, and a single SSC region, which was similar to that of most vascular plants $[38,39]$. The total length of the chloroplast genomes in this study ranged from 160,124 bp ( $T$. hemsleyanum from Jiangxi) to $161,430 \mathrm{bp}$ (A. japonica), that was consistent with the reported cp genomes from multiple other plants in Vitaceae, such as $A$. grossedentata $(162,147 \mathrm{bp})$ and $V$. davidii $(160,950 \mathrm{bp})[40,41]$. In spite of highly conserved cp genomes of angiosperms, gene loss and gain events continually occurred in certain species [42]. In our observations, the ycf15 gene was only encoded uniquely in $T$. hemsleyanum and $A$. japonica, while it absented in T. planicaule and $V$. vinifera (Table 2). According to the previous studies regarding 10 Chinese wild Vitis species [43], the uniform loss of $y c f 15$ gene was indicated as one of the essential characteristics in Vitis plants. However, other reports from Ampelopsis species showed that the ycf15 gene was disabled in A. brevipedunculata [44], and A. humulifolia [45]. These results further suggested relative complex regulatory functions of $y c f 15$ gene in the evolutionary of Vitaceae plants. Additionally, the trnS-GCU gene was merely encoded by T. hemsleyanum, while trnG-GCC, trnG-UCC and trnV-GAU were solely encoded by other three Vitaceae species, evidently reflecting the uniqueness of $T$. hemsleyanum in the family of Vitaceae. It is worth noting that the four special tRNA genes were all encoded by Ampelopsis brevipedunculata [44], resulting in a total of 31 distinct tRNA genes. The above results indicated that gene losses are not always dependable markers for phylogenetic relationships and further explorations focused on gene functions ought to be implemented and investigated urgently. With regards to the GC contents, chloroplasts from genus Tetrastigma (37.49-37.52\%) showed slightly higher ratio than that of most Ampelopsis species (37.33-37.37\%) [44, 45] and Vitis species (37.05-37.40\%), except that of $V$. romanetii (38.28\%) exhibiting an unusually large genome in size (232,020 bp) [43]. It is common knowledge that GC base pair is more stable than the AT. Accordingly, the increase of GC content in Tetrastigma species could potentially improve the stability of chloroplasts, consequently contributing to their adaptation to some harsh growing environments such as rocks crevices.

The expansion and contraction of the IR boundary is one of the main driving forces of changes in chloroplast genome size [46]. Except for the rp/2 gene, there was no significant variation among the T. hemsleyanum chloroplasts from different regions. In addition, only slight IR expansions and contractions were found in every border of $T$. hemsleyanum chloroplasts, further confirming their conserved traits of IR boundary (Fig. 3). However, the analyzed results indicated the location and the intron number of $r p / 2$ gene were diverse in $T$. hemsleyanum cp genomes from different regions. The rp/2 gene of T. hemsleyanum cp genome from Sichuan, Fujian and Jiangxi T. hemsleyanum cp genomes showed one intron and located in IRA completely, which was consistent with the reports of previous researches on T. planicaule [47] as well as other Vitaceae species [45, 48]. Remarkably, $T$. hemsleyanum chloroplasts from Guangxi and Zhejiang provinces possessed two introns that located across the border of JLA, which was not common in Vitaceae plants (Fig. 3). Nevertheless, several lineages of dicotyledons, including Saxifragaceae and Convolvulaceae, have even been reported to lose the intron of $r p / 2$ gene independently [49], which was regarded as the main characteristic of core members of Caryophyllales [50]. Moreover, shrinkage and expansion of the IR boundary could also trigger the duplication of genes or the generation of pseudogenes in angiosperms chloroplast genome [51]. Among the analyzed Vitaceae species in this study, only the cp genome of V. vinifera possessed a pseudogene $y c f 1$ located in IRB region completely. The pseudogenizations of $y c f 1$ gene were also documented in other Vitis species [43]. However, the similar event was not observed in Ampelopsis and Tetrastigma plants. Previous studies have suggested that $y c f 1$, a well-known gene with the most variable sites in $\mathrm{cp}$ genome, could be a promising DNA barcode with better performance than these current universal barcodes [52]. Nevertheless, our results showed that ycf1 region was not the marked hotspot with the most variation sites during the cp genome comparison analysis among T. hemsleyanum plants and other four Vitaceae species. Therefore, the function of ycf1 gene and its specific role in phylogenetic relationship of Vitaceae need to be further elucidated with more $\mathrm{cp}$ genomes. 
The adaptive evolution of $\mathrm{cp}$ genome genes represented valuable tool for exploring the variation of gene function, structural change and evolutionary of species [53]. The pairwise Ka/Ks values have been extensively used as an efficacious indicator to reveal positive selection pressure and adaptive evolution rate of species [54]. For the protein-coding genes with RNA editing sites in T. hemsleyanum chloroplasts in our study, the majority (91.67\%) of $\mathrm{Ka} / \mathrm{Ks}$ values exhibited a range from 0 to 0.5 , which was in accordance with the previous researches on some Vitaceae plants [44]. The lowest Ka/Ks value ( 0 ) was observed within genes encoding subunits of photosystem II ( $p s b F$ and $p s b L$ ) and protease ( $c / p P)$. While the most salient Ka/Ks values happens on matK and rps16, which encoded maturase and small subunit of ribosome. The higher Ka/Ks values of matK and $r p s 16$ indicated that these two genes might be more sensitive to the environment changes. The combination approach of matK and rps 16 sequences has been successfully applied to distinguish the primitive species of Polygonatum species [55]. In addition, the matK gene was also considered to be necessary for the survival of plant cells, and its expression required seriously strict regulation to prevent pernicious effects [56]. The previous data have suggested that the evolution of matK region was considered as the fastest gene in chloroplast genome, providing a powerful marker for genetic classification of jewel orchid accessions in Vietnam [57]. Herein, the matK gene with high Ka/Ks value could also be conjectured to be potential for species identification and genetic relationship study among Vitaceae plants.

\subsection{Phylogenetic Analysis and Taxonomic Implications within Family Vitaceae}

The grape family Vitaceae is an economically important family of angiosperms containing 16 genera and about 950 species. The Phylogenetic analysis based on the chloroplast genome sequences strongly supported the position of the Vitaceae as the earliest diverging lineage of rosids [58]. In addition, the Vitaceae family could be divided into five tribes by both nuclear and chloroplast genomic data, including Ampelopsideae, Cayratieae, Cisseae, Parthenocisseae, and Viteae, respectively [59]. Our phylogenomic analysis recovered three well supported lineages within Vitaceae (Fig. 8) that correspond to the three tribes reported in previous study [37]. The evolutionary relationships constructed in our study were consistent with those of previous researches $[37,60,61]$, further confirming the advances of whole chloroplast genome analysis on the phylogenetic resolution in certain lineages. The molecular phylogenetic analysis of Vitaceae was initially investigated utilizing several plastid genes, including the $\operatorname{trn} L-F$, atpB-rbcL, $\operatorname{rn} C$ petN, trnH-psbA, spacer and rps16 intron [61, 62]. A phylogenetic tree of Vitaceae with 417 single-copy nuclear genes was reconstructed from transcriptomes of 15 Vitaceae species, providing robust support for the deep relationships of the grape family and indicating the phylogenetic utility of transcriptome data for plants over a time scale. [63]. Subsequently, the deep relationships of Vitaceae family was explored by skimming the chloroplast and mitochondrial genomes for 27 taxa, supporting the division of five major clades of the family: the Vitis-Ampelocissus clade, the Parthenocissus-Yua clade, the core Cissus clade, the Cayratia-Cyphostemma-Tetrastigma (CCT) clade, and the Ampelopsis-Rhoicissus clade [37, 64]. It is interesting to note that the plants of tribe Cayratieae, including genera Causonis, Cayratia, Cyphostemma, Pseudocayratia and Tetrastigma, exhibited larger genomes than other four tribes in grape family [65]. Specially, five chloroplast molecular markers were employed to the phylogeny and biogeography of Cayratia, confirming the monophyly of the СCT clade and further suggesting its geographical origin of continental Africa in the late Cretaceous [60]. Furthermore, phylogenetic relationships within 72 Tetrastigma species recognized six strongly supported clades on the basis of ten plastid DNA regions, which do not completely correspond to their geographical distributions [66]. Nevertheless, the genus of Cayratia has been proved to be divided into three branches, relating to their geographical distribution [67]. The genus of Cayratia in Africa formed an independent group, while the other two groups were consisted of samples from Asia and Australia, which reflected the great impacts of different geographical origins on phylogenetic classification [68]. In this study, we determined the complete cp genomes of $T$. hemsleyanum from five provinces and constructed a comparative analysis with representing plants from tribe Cayratieae, which enriched the genome database of $T$. hemsleyanum and provided the basic data for improving the phylogenetic relationships among Tetrastigma species with better resolution. However, the sample sources in our research were all from China in Asia. Studies including a wider sampling area should be carried out across the genus to further elucidate the deeper relationships within Tetrastigma and Cayratieae.

\subsection{Development of Molecular Markers for Efficient Species Classification in Vitaceae}

The complete chloroplast genomes have proven to be powerful tool to resolve evolutionary relationships among plant species, and provide valuable information for molecular species authentication [69]. Compared to the potential markers from nuclear genome, the chloroplast genome derived markers harbored rich gene copies in plants and sufficient inter-specific divergence coupled with low intra-specific variations [70]. The identified highly variable regions of $\mathrm{cp}$ genomes could be developed as efficient DNA barcodes and used extensively in rapid species identification and large-scale phylogenetic analysis [71, 72]. The previous reports have confirmed that seven DNA barcodes selected based on the basis of the cp genomes of Pterocarpus species displayed better discernibility when comparing with the universal barcodes of $r b c L, m a t K, t r n H-p s b A$ and $t r n L-F[73,74]$. In our study, a total of five hypervariable regions across four complete cp genomes of Vitaceae species were identified, of which four were intergenic regions (rps16-trnQ, psbM-trnD, psbZ-trnfM and ycf3-trnS) and one was protein coding gene ( $y c f 1)$ (Fig. 6A). In addition, the IR regions of Vitaceae plants exhibited significant lower sequence divergence than the SSC and LSC regions, which was consistent with the results from other plants [75]. It is a very common phenomenon in the cp genomes of most angiosperms, where the intergenic spacers contained more sequence variants than the coding genes. The developed molecular markers with higher inter-specific levels have been widely reported in other studies and exhibited excellent discriminating ability in investigating taxonomy and molecular phylogeny, such as Pulsatilla of Ranunculaceae [76] and Rhodiola of Crassulaceae [77]. It is worth noting that the three intergenic regions, rps16-trmQ [78], psbM-trnD [40] and psbZ-trnfM [79] found in our results were also reported to be candidate DNA barcodes for plant identification and phylogenetic relationships analysis in Echinacanthus, Myrtales and Zygophyllum species, respectively. The deep phylogenetic relationship within the tribe Cayratieae and family Vitaceae still remains uncertain due to high morphological similarity and less molecular data. Furthermore, the dried root from T. hemsleyanum bears a striking morphological resemblance to that from closely 
related species of Cayratieae and Vitaceae, which caused the emergence of adulterant and counterfeit drugs in the market and led to potential hazard to health and clinical efficiency. The molecular phylogeny based on the combination of three chloroplast markers suggested the split of genus Vitis into three clades and supported a relatively recent and intense gene flow in species from different regions [80]. The universal DNA barcode ITS2 was reported to distinguish $T$. hemsleyanum from its adulterants, providing an effective and accurate identification strategy for this endangered herb [12] However, we failed to sequence and amplify the ITS2 gene with the DNA template extracted from the processed roots of T. hemsleyanum, indicating the potential destruction on nuclear genomes during the processing of crude drugs (data not shown). The destroyed nuclear DNA brought the limitations of universal nuclear DNA barcode sequences for distinguishing processed medicinal plants. Contrast with the nuclear barcode ITS2, the developed chloroplast genome markers of $t r n L$ and $t r n N$ could be successfully amplified with the genomic DNA from the dried root of $T$. hemsleyanum, suggesting the chloroplast genome was more stable than the nuclear DNA during the processing of medicinal plant. Therefore, the identified mutational hotspots regions and according primer set were believed to help to distinguish the taxa in the genus level, which would provide a credible approach to identify related species and assess the interspecific phylogenetic relationships among Vitaceae plants.

The hypervariable region worthy of special attention was $y c f 1$, which exhibited the highest Pi value among the coding genes in this study (Fig. $6 \mathrm{~A}$ ). The DNA barcode of $y c f 1$ has been confirmed the excellent ability in identification of Fritillaria species [81] and phylogeny reconstruction of Primula species [82]. The comparative analysis of the $y c f 1$ gene in four Vitaceae plants revealed that fewer SNP sites and Gaps were shown between $T$. hemsleyanum and T. planicaule while more variations were presented between A. japonica and $V$. vinifera (Table 6). These results indicated that the ycf1 was more reliable for the research on phylogenetic relationships in Vitaceae plants than the species identification of related plants within Tetrastigma. In addition, the PCR amplified length of the currently determined three hypervariable regions of $p s b M$-trnD, psbZ-trnfM and $y c f 1$ were less than $1000 \mathrm{bp}$, which could result in the high success rates of amplification and sequencing. Therefore, these three diversity regions presented candidate barcoding sequence, which might be helpful to plant identification, systematic investigation of Vitaceae and evaluating the phylogenetic relationships among the tribe of Cayratieae.

\subsection{Geographical Origin Discrimination Strategy for Medicinal Plants}

The quality of medicinal plants depends on various factors with significant contributions of genetic impacts and geographic location. The development of geo-authentic Chinese medicinal material was closely associated with cultivated outstanding genetic mutants and eligible local environmental impacts, leading to the production of famous crud drugs with higher quality and price in the market [83]. With the increasing global demand for plant medicines, great numbers of medicinal species have been cultivated in different areas to generate multiple genetic populations with similar morphological features [84]. Due to the significant advantages of genuine medicinal material from geo-authentic producing areas, it was more likely to be adulterated with lower-price counterparts from other different regions [85]. However, the geographical origin of herbal medicines has been indicated as a crucial factor influencing the quality and potential treatment efficacy of the medicinal materials, which could be attributed to the variations in the environmental conditions and genetic reasons [86]. Zhang et al. [87] confirmed that there were obvious differences in chemical components of dandelions from four different geographical regions by metabolomics analysis, especially in phenolic compounds. To ensure the health benefits and clinical effectiveness of herbal medicine to consumers, it is necessary to develop strategies for the recognition of geographic origin crud drugs. Since the obvious differences on the content of chemical constituent and plant genetic populations, several analytical approaches of DNA and chromatographic analysis have been extensively applied to determine the geographical origin of medicinal plants and foods [88]. The HPLC similarity analysis and content of alkaloids was indicated as valuable tools for differentiating the geographical origin of the Fuzi samples [89]. The excitation-emission matrix fluorescence and chemometric strategies have also been considered as promising methods for distinguishing the geographical origin of Gastrodia elata [90]. Compared with the chemotaxonomical investigations, the DNA analysis based on either unique sequence regions or DNA polymorphism from genetic markers represented alternative approaches to identify plant populations and authenticate plant species. The amplification of unique gene fragment exhibited a rapid and easy method to identify the geographical authenticity of Scrophularia ningpoensis [67]. The SSR markers were indicated as suitable tool for assessing genetic diversity and population structure of spinach germplasm, which clearly separated the accessions with different geographical origins [91]. Recently, the combination of DNA molecular markers and chemical analytical techniques has been successfully used to distinguish the geographical origins of traditional Chinese medicines. The integration of microsatellite markers and chemical analysis could discriminate the Moutan Cortex from different sources and geographical origins [92]. The ISSR fingerprinting combined with FTIR spectrum analysis established a rapid and efficient approach to determine the Cassia tora populations with different ecogeographical origins [93]. The development of efficient geographical tracing system of medicinal plants would significantly contribute to the protection of genuine plant genetic resources and improvement for quality control of herbal drugs.

Since the significantly differences on the crude drug quality and clinical efficiency of $T$. hemsleyanum from different regions in China, a variety of identification approaches have been reported for discriminating geographical origins of $T$. hemsleyanum, including determining strategies based on macroscopic analysis, spectroscopic technology, chromatographic fingerprint and bio-activity evaluation. Previous study has reported that the root tuber of T. hemsleyanum from Zhejiang and Guangxi province could be successfully distinguished by analyzing the external characteristics of tuber and the typical micro-structures of powder, thus providing an intuitive and simple approach for plant origin determination [94]. However, pharmacognosy-based identification cannot accurately identify the processed decoction pieces of $T$. hemsleyanum from the above two aspects. Li et al. [95] effectively distinguished the T. hemsleyanum samples from Zhejiang, Yunnan and Guizhou Province using a combined identification approach based on HPLC fingerprints and the random forest (RF) algorithm analysis. Machine learning algorithm has been widely used for spectral data processing to discriminate medicinal herbs from different habitats. A dual-mode microscopic hyperspectral imager (DMHI) system has been developed using the combined dataset of RMHI and FMHI modes for hyperspectral detection of the origins and varieties of T. hemsleyanum, obtaining a

Page $15 / 24$ 
prediction accuracy as high as $97.5 \%$ of both origins and varieties [96]. Besides, the near-infrared spectroscopy (NIRS) combined with deep learning models also exhibited potential capability to distinguish the medicinal plant T. hemsleyanum among different origins [97]. However, the processing of crude drugs and growth years and harvesting seasons of medicinal materials can significantly affect the geographical origin identification methods based on chemical contents and compositions, thus generating inaccurate results. Furthermore, deep learning is complex, time-consuming, and associated with a low signal-to-noise ratio (SNR), instability, and spectrum peaks overlap, thus limiting its application. In contrast, the origin discrimination approaches based on DNA markers could target the direct carrier of genetic information in plant populations, thus providing an alternative strategy with higher stability than those based on chemical analysis. Therefore, this study provides an efficient method for distinguishing the geographical origins of $T$. hemsleyanum based on DNA barcodes from the $\mathrm{cp}$ genome from different regions. The developed specific DNA barcodes and their combination divided the $21 \mathrm{~T}$. hemsleyanum samples from six provinces into three haplotypes and successfully classified a sample from Genbank into clade区. The specie of T. hemsleyanum exhibited genetic patterns characteristic of long-term separation in multiple refugia and lower levels of interpopulation gene flow, indicating that the genetic population divergence largely driven by mutation or drift, further supporting the genetic stability of T. hemsleyanum samples in this study [1]. Previous study on lineage diversification reported that $T$. hemsleyanum was consisted of two major cpDNA lineages, Southwest (SW) and Central-South-East (CSE) China, consistent with our grouping results [98]. Herein, the T. hemsleyanum samples from CSE China were divided into two groups, while those from Sichuan Province in SW China were grouped in one cluster. Furthermore, the method based on universal DNA barcode ITS2 suggested that T. hemsleyanum from Zhejiang province had a unique genetic status, exhibiting potentiality for the plant population and geographical origin discriminating between Zhejiang and other provinces [12]. Besides the DNA molecular barcoding strategy, other molecular markers of RAPD and SRAP also displayed the potential capacity in determining the geographical origins of $T$. hemsleyanum. For instance, Yin et al. [99] have sifted out 10 pairs of RAPD primers for PCR amplification of 64 samples of T. hemsleyanum from 14 provinces in China. They revealed abundant genetic diversities of T. hemsleyanum germplasm resources and significant complexity of geographical distribution. However, the RAPD cluster analysis was inconsistent with the geographical distance of the provenance, requiring further revisions for primers for geographical origin determination. Notably, SRAP markers divided the same 64 T. hemsleyanum samples into nine groups, which exhibited certain different results with that from RAPD analysis [100]. Herein, the combination of $t r n L+\operatorname{trnN}$ barcode divided the T. hemsleyanum plants into three groups, thus effectively identifying the genetic populations of T. hemsleyanum samples from Zhejiang and Sichuan provinces. However, our results failed to distinguish the strains in each of other provinces concretely, generating a clade with $T$. hemsleyanum samples from Zhejiang, Jiangxi, Fujian, Guangxi, Guangdong and Genbank database. Consortium for the Barcode of Life [101] has confirmed that combining barcodes has a better identification efficiency than a single barcode. Therefore, the combination mode of universal barcodes of $t r n H-p s b A$, ITS2, matK and specific developed barcodes of $t r n L$ and $t r n N$ would be a promising approach for the accurate traceability of $T$. hemsleyanum from different regions. Moreover, the distribution of repeats in the genome could infer highly polymorphic regions in the cp genome [102].

SSRs have become a new molecular marker technology and extensively applicated in plants genetic diversity, gene mapping and variety identification with the characteristics of strong polymorphism, co-dominance, high universality and good stability [103]. The distribution of chloroplast SSR characteristics exhibited taxon specificity in Cyatheaceae species, which provided valuable phylogenic information at the genus level [104]. Furthermore, the SSR primers designed based on different Gracilaria tenuistipitata chloroplast genomes from various regions could separate the samples into two main geographical regions, which significantly contributed to the mass cultivation of seaweeds with high economic potential [105]. The developed program, ChloroSSRdb focused on the application of chloroplastic SSRs from Viridiplantae, thus providing useful resources in developing genetic markers and phylogenetic analysis [106]. The ISSR molecular markers divided the germplasm resources of $T$. hemsleyanum from the main distribution areas of China into 4 Clades, among which Zhejiang samples were all clustered in the Clade $\otimes$ [107]. Another report showed that ISSR and SRAP markers could cluster the wild accessions of T. hemsleyanum into four groups (similarity coefficient level, 0.75) [14]. All the wild populations from Zhejiang were highly distinct for ISSR polymorphism and formed a separate cluster, while those from the other three clusters consisted of T. hemsleyanum samples from Guangxi, Jiangxi and Hunan. This study also found abundant SSRs in the cp genomes of $T$. hemsleyanum plants. Furthermore, significant differences were identified on SSR numbers and types in the T. hemsleyanum cp genomes among five samples from different regions, mainly distributed in quantities of mononucleotide repeats and types of repeat unit (Fig. 4A). Meanwhile, a comparison among four Vitaceae species including T. hemsleyanum from Jiangxi has revealed great discrimination in SSR counts and types between different tribes, such as tribe Cayratieae, Ampelopsideae and Viteae, suggesting the potentiality of SSRs in species discrimination and classification (Fig. 4B). However, most of current studies focused on the interindividual genetic variation of $T$. hemsleyanum, thus limiting the use of SSR markers in determining the genetic populations from different regions. Therefore, the SSRs fingerprints should be systematically constructed for geographical origin identification of $T$. hemsleyanum based on the present comparative analysis of $\mathrm{cp}$ genomes from different regions.

\section{Materials And Methods}

\subsection{Plant Material and DNA Extraction}

The plant materials of T. hemsleyanum were collected from six different provinces in China (Supplementary Table 1) and identified by Dr. Yuqing Ge of Zhejiang Chinese Medical University. The plant specimens were deposited at Medicinal Herbarium Center of Zhejiang Chinese Medical University (https://yxy.zcmu.edu.cn, Herbarium Code: MHCZCMU, collector: Rubin Cheng, biothcheng@hotmail.com). The detailed voucher numbers of $T$. hemsleyanum with different geographical origins were listed in Table S1. Total genomic DNA was extracted from the fresh and healthy leaves of T. hemsleyanum using a modified cetyltrimethylammonium brofmide (CTAB) method [108]. The final DNA integrity and concentration were assessed by electrophoresis on 1.0\% agarose gel and Nanodrop 2000 Spectrophotometer (Thermo Fisher Scientific, United States).

Page $16 / 24$ 


\subsection{Sequencing, Genome Assembly and Annotation}

The paired-end (150 bp) sequencing of the DNA libraries was conducted on the Illumina HiSeq 2500 platform, generating about 2.4 GB of raw data for each sample. Then the quality of paired-end Illumina reads was assessed with FastQC, and the low-quality reads were removed using Fastp. The filtered reads were assembled de novo using metaSPAdes with the complete cp genome of T. hemsleyanum (NC_029339) as reference and the proteincoding genes, mRNA genes, tRNA genes were annotated by GeSeq annotation tool [109]. The CPGAVAS2 software also used to annotate proteincoding genes [110]. BLAST was further used to correct the annotation of chloroplast genome manually. The circular chloroplast genome map of $T$. hemsleyanum collected from Jiangxi Province were drawn by OrganellarGenomeDRAW (OGDRAW) tool [111]. Finally, the fully annotated cp genomes were deposited at the GenBank database (Supplementary Table 1).

\subsection{Comparative Analysis of Chloroplast Genomes and Identification of Hypervariable Regions}

MEGA 7.0 [112] was used to analyze the genome feature and Codon W software was used to investigate the distribution of codon usage using the RSCU value [113]. The IR/SC boundary locations in five samples of $T$. hemsleyanum and three representative Vitaceae species were compared using IR scope [114]. As for the repeats analysis, long repeats of four different type (forward (F), palindromic (P), reverse (R), and complementary (C)) were identified by REPuter, with hamming distance 3, minimal repeats 30 and maximum computed repeats 50 [115]. Simple sequence repeats in Vitaceae species were detected by MISA, setting parameters as 10 for mononucleotide SSRs, 5 for dinucleotide SSRs, 4 for trinucleotide SSRs, 3 each for tetranucleotide, pentanucleotide and hexanucleotide SSRs [116]. To predict the number of RNA editing sites, the PREP-Cp program was employed with a cutoff value 0.8 [117]. For the identification of hypervariable regions within five samples of $T$. hemsleyanum and among four representative species of Vitaceae, we aligned cp genome sequences using MAFFT [118] and evaluated the sequence divergence among Vitaceae species through a sliding window analysis in DnaSP v6 [119]. The parameters of sliding window analysis were set as window length for 800 sites and the step size of 200 sites.

\section{4. $\mathrm{Ka} / \mathrm{Ks}$ and Positive Selection Analyses}

In order to analyzed the Ka and Ks substitution rates and Ka/Ks ratio, Melaleuca cajuputi was compared with T. hemsleyanum (Jiangxi Province) in 24 protein coding genes. The alignment was carried out by MAFFT v7.037b [118], and the calculation of the value of Ka/Ks was implemented by DnaSP v6 [119].

\subsection{Primier Design and PCR Amplification}

Based on conserved nucleotide sequences at both ends of mutation hotspots, 5 pairs of specific primers were designed by Primer Premier 5 (Supplementary Table 2). PCR was performed in 50- $\mu \mathrm{L}$ reactions consisting of $3 \mu \mathrm{L}$ of genomic DNA, $5 \mu \mathrm{L}$ of dNTP Mix and 10x LA PCR Buffer, $2.5 \mu \mathrm{L}$ of forward and reverse primers ( $25 \mu \mathrm{mol} / \mathrm{L}), 0.5 \mu \mathrm{L}$ of LA Taq (Takara) and ddH2O supplemented to $50 \mu \mathrm{L}$. PCR amplification was carried out by Veriti Thermal Cycler (Applied Biosystems) with the following program: 5 minutes at $94^{\circ} \mathrm{C}$ for initial denaturation; denaturation $94^{\circ} \mathrm{C}, 45$ seconds; 33 cycles consisting, annealing temperature $53^{\circ} \mathrm{C}-58^{\circ} \mathrm{C}, 30$ seconds, extension temperature $72^{\circ} \mathrm{C}, 45$ seconds, final 10 minutes extension at $72^{\circ} \mathrm{C}, 4^{\circ} \mathrm{C}$ low temperature save. PCR products were examined by electrophoresis on 1.0\% Agarose Gel and visualized with 4S GelRed Nucleic Acid Stain (Sangon Biotech, China). Finally, optimum annealing temperature of $t r n L$ and $t r n N$ sequences was determined as $56^{\circ} \mathrm{C}$. The synthesis of primers and Sanger sequencing were conducted by Sangon Biotechnology and Shanghai Sunny Biotechnology Co., Ltd., respectively.

\subsection{Multiple Sequence alignment and data analysis}

The sequencing results were performed using BioEdit 7.0 [120] and aligned by MAFFT 7.0 [118] to quantify the sequence length and base composition. To count the variant information, the aligned sequences were analyzed by MEGA 7 [112]. Based on the single and combination sequences, the intraspecific genetic distance of T. hemsleyanum between Jiangxi Province and other regions, as well as the interspecific genetic distance among representative species of Vitaceae were calculated using MEGA 7 with Kimura 2-parameter (K2P) distance model.

\subsection{Phylogenetic Analysis and Effectiveness of marker Discriminatory}

The maximum likelihood (ML) tree and maximum parsimony (MP) tree were constructed using 70 conservative protein-coding genes of five $T$. hemsleyanum complete $\mathrm{cp}$ genomes sequenced in this study and 10 additional publicly available sequences that we downloaded from the NCBI. Among these 15 species, Melaleuca alternifolia and Melaleuca cajuputi were chosen as outgroups. The ML tree was constructed based on the K2P model with 500 bootstrap replications. Similarly, MP tree was obtained from MEGA 7 [112] under default parameters with 500 bootstrap replications. Based on the K2P distance model, the single and combination $\operatorname{trn} L$ or $\operatorname{trnN}$ sequences of Vitaceae species were used to construct the NJ trees with 1000 bootstrap replications.

\section{Conclusions}

This study provides 5 complete chloroplast genome sequences of Tetrastigma hemsleyanum with different geographical origins in China, and presents a comparative analysis of $\mathrm{cp}$ genomes with other representing species from family Vitaceae. The chloroplast genome structure of $T$. hemsleyanum samples from different regions and other Vitaceae plants was highly conserved. However, IR expansion and contraction was observed among $\mathrm{cp}$ genomes of $T$. hemsleyanum from different areas, resulting in $\mathrm{cp}$ genomes of different sizes. In addition, significant differences in SSR 
types and numbers were identified in the cp genomes of different $T$. hemsleyanum samples as well as Vitaceae species, providing valuable genetic information for the development of species identification strategy and geographical origin determining system. Phylogenetic analysis revealed the five T. hemsleyanum plants clustered together to form a stable monophyletic group, exhibiting sister relationship with $T$. planicaule to compose the tribe of Cayratieae. A total of five highly variable regions with significant differences between $T$. hemsleyanum and other Vitaceae species were identified and may be applied as potential markers for species identification and further phylogenetic relationship analysis in family of Vitaceae. Furthermore, the cp molecular markers of $t r n L$ and $t r n N$ were successfully built based on the hotspots among $T$. hemsleyanum cp genomes with different regions. The combination of $t r n L$ and $t r n N$ could divide the $T$. hemsleyanum plants from six different regions into three genetic groups, providing potential molecular tool for the geographical origin discrimination of T. hemsleyanum in China. These results obtained in this study would contribute to the understanding of phylogenetic relationship and systematic evolution of Vitaceae plants, and provide valuable molecular approaches to discriminate the geographical origins of $T$. hemsleyanum and accurately distinguish the original plants from its adulterants.

\section{Abbreviations}

BLAST: Basic local alignment search tool; cp: chloroplast; DNA: Deoxyribonucleic acid; IR: Inverted repeat; LSC: Large single copy region; SSC: Small single copy region; SSR: Simple sequence repeats.

\section{Declarations}

\section{Ethics approval and consent to participate}

Since the Basic Public Welfare Research Project of Zhejiang Province, Dr. Rubin Cheng has obtained the permission by Zhejiang Chinese Medical University to collect plant species of Tetrastigmahemsleyanum from different areas of China for further molecular study. The complete chloroplast genome analysis and subsequent DNA barcoding markers were applied to classify the geographical origins of T. hemsleyanumfrom Zhejiang, Jiangxi, Fujian, Sichuan, Guangxi and Guangdong province. The plant material collection and experimental research were conducted according to the Plant Protection and Regulation of Zhejiang Chinese Medical University.

\section{Consent for publication}

Not applicable.

\section{Availability of data and materials}

The datasets generated for this study can be found in National Center for Biotechnology Information (NCBI) under the accession numbers: MW375707-MW375711; MZ995433-MZ995472; OK058531-OK058532.

\section{Competing interests}

The authors declare that they have no competing interest.

\section{Funding}

This research was funded by the Basic Public Welfare Research Project of Zhejiang Province, grant number LGF22H280005 and the Research Project of Zhejiang Chinese Medical University, grant number 2021JKZKTS020B.

\section{Author contributions}

SD, YG and RC conceived and designed the work. MZ, JZ and RC contributed to the resource sampling. SD, MJ and QW performed the experiments. SD, $\mathrm{JZ}$ and YG analyzed the data and wrote the main manuscript, prepared the figures and tables, RC and YG supervised the project. All authors have read and approved the final manuscript.

\section{Acknowledgments}

We are grateful to Shanhua Zhang, Fangfang Ma and Shuisheng Yu for sample collection and field assistance. We appreciate the great help and experimental support from the Public Platform of Pharmaceutical Research Center, Academy of Chinese Medical Science, Zhejiang Chinese Medical University.

\section{References}

1. Wang YH,Jiang WM, Comes HP, HuFS,QiuYX, Fu CX.Molecular phylogeography and ecological niche modelling of a widespread herbaceous climber, Tetrastigmahemsleyanum (Vitaceae): insights into Plio-Pleistocene range dynamics of evergreen forest in subtropical China. New Phytol. 2015; 206: 852-67. 
2. Zhu RY, Xu XF, Ying JL, Cao G, WuX. The phytochemistry, pharmacology, and quality control of Tetrastigmahemsleyanum Diels \&Gilg in China: A Review. Front. Pharmacol. 2020;11: 550497.

3. Hu WY, Zheng YJ, Xia PG, Liang ZS. The research progresses and future prospects of Tetrastigmahemsleyanum Diels et Gilg: A valuable Chinese herbal medicine. J. Ethnopharmacol. 2021;271: 113866.

4. Qiu MC, Zhou ZD, Yang ZJ.Total flavonoids from Tetrastigmahemsleyanum Diels induce apoptosis of breast cancer cells through the MAPK pathway. Chin. J. Clin. Pharmacol. 2019;35: 305963.

5. Li YL, Feng XY, ZhangYR, Wang YX, YuX, Jia RY, et al. Dietary flavone from the Tetrastigmahemsleyanum vine triggers human lung adenocarcinoma apoptosis via autophagy. Food \& function.2020;11: 9776-88.

6. Chu Q, Chen W, JiaRY, Ye X, Li YL, Liu YY,et al. Tetrastigmahemsleyanum leaves extract against acrylamide-induced toxicity in HepG2 cells and Caenorhabditis elegans. J. Hazard. Mater. 2020;393: 122364.

7. Zhou, F.-M.; Chen, Y.-C.;Jin, C.-Y; Qian, C.-D.; Zhu, B.-Q.; Zhou, Y.; Ding, Z.-S.; Wang, Y.-Q. Polysaccharide isolated from Tetrastigmahemsleyanumactivates TLR4 in macrophage cell lines and enhances immune responses in OVA-Immunized and LLC-Bearing mouse models. Pharmacol.2021, 12, 609059.

8. Sun Y, Li HY, Hu JN, Li J, FanYW, Liu XR, et al. Qualitative and quantitative analysis of phenolics in Tetrastigmahemsleyanum and their antioxidant and antiproliferative activities. J Agric Food Chem. 2013;61: 10507-15.

9. Ji T, JiWW, Wang J, Chen HJ, Peng X, Cheng KJ, et al. A comprehensive review on traditional uses, chemical compositions, pharmacology properties and toxicology of Tetrastigmahemsleyanum. J Ethnopharmacol. 2021;264: 113247.

10. Chen PT, Chen LQ, Wen J. The first phylogenetic analysis of Tetrastigma(Miq.) Planch., the host of Rafflesiaceae. Taxon. $2011 ; 60: 499-512$.

11. Pelser PB,Nickrent DL, Barcelona JF. Untangling a vine and its parasite: Host specificity of Philippine Rafflesia (Rafflesiaceae). Taxon.2016;65: 739-58.

12. Peng X, Wu XR, Ji QY, Yang RK, LiYL. Molecular authentication of Tetrastigmahemsleyanum from its adulterant species using ISSR, CAPS, and ITS2 barcode. Mol Biol Rep. 2016;43: 785-94.

13. Liu, Z.-G.; Li, M.-Z.; Tao, Y.-D.; Olsen, R. Multivariate statistical and comparison analysis of chemical constituents in Arenaria kansuensis from different regions in Qinghai-Tibet Plateau. Phytochem Analysis.2021; 32: 3025.

14. Peng X, Ji QY, Fan SW, Zhang,YJ, Zhang JJ. Genetic diversity in populations of the endangered medicinal plant Tetrastigmahemsleyanumrevealed by ISSR and SRAP markers: implications for conservation. Genet Resour Crop Evol. 2015;62: 1069-78.

15. Li YJ, Fu JE, WeiSG, Pan LM. Comparison of total flavonoids content of Tetrastigmahemsleyanum from different regions and its Inhibition rate on Proliferation of HepG2 Cells.J Zhejiang Chin Med Univ.2014;24: 40-1.

16. Yin SY, Cui HR, Zhang L, Yan JR, Qian LH. Transcriptome and metabolome integrated analysis of two ecotypes of Tetrastigmahemsleyanum reveals candidate genes involved in chlorogenic acid accumulation. Plants.2021; 10: 1288.

17. Gong WX, Liu T, Zhou ZD, Wu DX. Shu XL,Xiong HZ. Physicochemical characterizations of starches isolated from Tetrastigmahemsleyanum Diels et Gilg.Int J Biol Macromol. 2021;183: 1540-7.

18. Daniell H, Chan HT,Pasoreck EK. Vaccination via chloroplast genetics: affordable protein drugs for the prevention and treatment of inherited or infectious human diseases.Annu Rev Genet. 2016;50: 595-618.

19. Lee SR, Kim K, Lee BY, Lim CE. Complete chloroplast genomes of all six Hosta species occurring in Korea: molecular structures, comparative, and phylogenetic analyses. BMC Genomics.2019;20: 833.

20. Shahzadi I, Abdullah, Mehmood F, Ali Z, Ahmed I, Mirza B. Chloroplast genome sequences of Artemisia maritima and Artemisia absinthium: Comparative analyses, mutational hotspots in genus Artemisia and phylogeny in family Asteraceae. Genomics.2020;112: 1454-63.

21. Iram, S, Hayat,M.Q, Tahir, M, Gul, A, Abdullah; Ahmed, I. Chloroplast genome sequence of Artemisia scoparia: comparative analyses and screening of mutational hotspots. Plants2019, 8, 476. doi: 10.3390/plants8110476.

22. Wu ZQ, Song G. The phylogeny of the BEP clade in grasses revisited: Evidence from the whole-genome sequences of chloroplasts. Mol PhylogenetEvol.2012; 62: 573-8.

23. Luo C, Huang WL, Sun HY,Yer HY, Li XY, Li Y,et al. Comparative chloroplast genome analysis of Impatiens species (Balsaminaceae) in the karst area of China: insights into genome evolution and phylogenomic implications. BMC Genomics.2021;22: 571.

24. Gu CH, Ma L, Wu ZQ, Chen K, Wang YX. Comparative analyses of chloroplast genomes from 22 Lythraceae species: inferences for phylogenetic relationships and genome evolution within Myrtales. BMC Plant Biol. 2019;19: 281.

25. Zhang YX,laffaldano BJ, Zhuang XF,Cardina J, Cornish K. Chloroplast genome resources and molecular markers differentiate rubber dandelion species from weedy relatives. BMC Plant Biol.2017;17: 34.

26. Wang AS, Wu HW, Zhu XC, Lin JM. Species identification of Conyza bonariensis assisted by chloroplast genome sequencing. Front Genet.2018;9: 374.

27. $\mathrm{Ng} \mathrm{CH}, \mathrm{Ng} \mathrm{KKS}$, Lee SL,Tnah LH, Lee CT, Zakaria N. A geographical traceability system for Merbau (Intsiapalembanica), an important timber species from peninsular Malaysia. Forensic Sci Int Genet. 2020;44: 102188. 
28. Fang HL, Guo QS, Shen HJ, Shao QS. Phylogeography of Chrysanthemum indicum (compositae) in China based on trnL-F sequences. Biochem. Syst. Ecol. 2010, 38, 1204-1211.

29. Liu HB, Lu YZ, Lan BL, Xu JC. Codon usage by chloroplast gene is bias in Hemipteleadavidii. Journal of Genetics. 2020;499: 1-11.

30. Palidwor GA, Perkins TJ, Xia XH. A general model of codon bias due to GC mutational bias.PLoS One.2010;5: e13431.

31. Plotkin JB, Kudla G. Synonymous but not the same: the causes and consequences of codon bias. Nat Rev Genet. 2011;12: 43-2.

32. Xu XD, Wang D. Comparative chloroplast genomics of corydalis species (Papaveraceae): evolutionary perspectives on their unusual largescale rearrangements. Front Plant Sci. 2021;11: 600354.

33. Lee J, Kang Y, Shin SC, Park H, Lee H. Combined analysis of the chloroplast genome and transcriptome of the Antarctic vascular plant Deschampsiaplaceantarctica Desv. PLoS One. 2014;9: e92501.

34. Li B, Lin F, Huang P, Guo W, Zheng Y. Development of nuclear SSR and chloroplast genome markers in diverse Liriodendron chinense germplasm based on low-coverage whole genome sequencing. Biol Res. 2020;53: 21.

35. Qian J, Song JY, Gao HH, Zhu YJ, Xu J, Pang XH,et al. The complete chloroplast genome sequence of the medicinal plant Salvia miltiorrhiza. PLoS One.2013;8: e57607.

36. Wang MX, Liu H, Ge LQ, Xing GW, Wang M, Song WN, et al. Identification and analysis of RNA editing sites in the chloroplast transcripts of Aegilops tauschii Genes. 2016;8: 13.

37. Wen J, Lu LM, NieZL, Liu XQ, Zhang N, Ickert-Bond SM, et al. A new phylogenetic tribal classification of the grape family (vitaceae).J Syst Evol. 2018;56: 262-72.

38. Tonti-Filippini J,Nevill PG, Dixon K, Small I. What can we do with 1000 plastid genomes?Plant J. 2017;90: 808-18.

39. Wicke S,Schneeweiss GM,Depamphilis CW, Müller KF,Quandt D. The evolution of the plastid chromosome in land plants: gene content, gene order, gene function. Plant Mol Biol.2011;76: 273-97.

40. Gu L, Zhang N, Feng C, Yi Y, Yu ZW. The complete chloroplast genome of Ampelopsis grossedentata(Hand-Mazz.) W. T. Wang (Family: Vitaceae) and its phylogenetic analysis. Mitochondrial DNA B Resour. 2020;5: 2423-4.

41. Tian QY, Fu PN, Wu W, Li RF,Koleva L, Lu J, et al.The complete chloroplast genome sequence of Vitis davidiFoex strain 'SJTU003'. Mitochondrial DNA B Resour.2019;4: 3370-1.

42. Millen R, Olmstead R, Adams K, Palmer J, Lao N, Heggie L,et al. Many parallel losses of infA from chloroplast DNA during angiosperm evolution with multiple independent transfers to the nucleus.Plant Cell.2001;13:645-58.

43. Xu GY, Xu WR. Complete chloroplast genomes of Chinese wild-growing Vitis species: molecular structures and comparative and adaptive radiation analysis. Protoplasma.2021; 258: 559-71.

44. Raman G, Park SJ. The Complete Chloroplast Genome Sequence of Ampelopsis: Gene Organization, Comparative Analysis, and Phylogenetic Relationships to Other Angiosperms.Front Plant Sci. 2016;7: 341.

45. Yu XL, Tan W, Zhang HY, Gao H, Wang WX, Tian XX. Complete Chloroplast Genomes of Ampelopsis humulifoliaand Ampelopsis japonica: Molecular Structure, Comparative Analysis, and Phylogenetic Analysis. Plants (Basel).2019;8: 410.

46. He L, Qian J, Li X, Sun Z, Xu X, Chen S. Complete chloroplast genome of medicinal plant Lonicera japonica: genome rearrangement, intron gain and loss, and implications for phylogenetic studies. Molecules. 2017;22: 249.

47. Huang X, Zhou QH, Qin CM, Mao C, Sun KD, Qin B,et al. The complete chloroplast genome of Tetrastigmaplanicaule one important folk medicinal plant in China. Mitochondrial DNA B Resour.2021;6: 1745-6.

48. Xiang J, Wei LZ, Cheng JH, Li MS, Cui PF, Wu J. The complete chloroplast genome sequence of Vitis champinii. Mitochondrial DNA B Resour. 2020;5: 2855-6.

49. Downie SR, Olmstead RG,Zurawski G,Soltis DE, Soltis PS, Watson JC,et al. Six independent losses of the chloroplast DNA rp/2 intron in dicotyledons: molecular and phylogenetic implications. Evolution. 1991;45: 1245-59.

50. Logacheva MD,Samigullin TH, Dhingra A,Penin AA. Comparative chloroplast genomics and phylogenetics of Fagopyrum esculentum ancestrale -a wild ancestor of cultivated buckwheat. BMC Plant Biol. 2008;8: 59.

51. Abdullah, Mehmood F,Shahzadi I, Waseem S, Mirza B, Ahmed I,et al. Chloroplast genome of Hibiscus rosa sinensis(Malvaceae): comparative analyses and identification of mutational hotspots. Genomics. 2019;112: 581-91.

52. Dong WP, Xu C, Li CH, Sun JH,ZuoYJ, Shi S,et al.ycf1, the most promising plastid DNA barcode of land plants. Sci Rep. 2015;5: 8348.

53. Nei M, Kumar S. Molecular evolution and phylogenetics. 2000, USA: Oxford University Press.

54. Yang Z, Nielsen R. Estimating synonymous and nonsynonymous substitution rates under realistic evolutionary models. Mol Biol Evol.2000;17: 3243.

55. Shen XX, Sun J,Wang ZA.Polygonatum medicinal plants based on chloroplast matKand rps16 Modern Chinese Medicine.2021;23: 275-9+325.

56. Qu YJ,Legen J, Arndt J, Henkel S, Hoppe G,Thieme C,et al. Ectopic Transplastomic Expression of a Synthetic MatK Gene Leads to CotyledonSpecific Leaf Variegation. Front Plant Sci.2018;9: 1453. 
57. Ho VT, TranTKP, Vu TTT,Widiarsih S. Comparison of matKand rbcL DNA barcodes for genetic classification of jewel orchid accessions in Vietnam. J Genet EngBiotechnol. 2021;19: 93.

58. Jansen RK,Kaittanis C,Saski C, Lee SB, Tomkins J,Alverson AJ, et al. Phylogenetic analyses of Vitis (Vitaceae) based on complete chloroplast genome sequences: effects of taxon sampling and phylogenetic methods on resolving relationships among rosids. BMC Evol Biol. $2006 ; 6: 32$.

59. Ma ZY,NieZL, Ren C, LiuXQ, Zimmer EA. Wen J. Phylogenomic relationships and character evolution of the grape family (Vitaceae). Mol PhylogenetEvol. 2021;154: 106948.

60. Lu LM, Cox CJ, Mathews S, Wang W, Wen J, Chen ZD. Optimal data partitioning, multispecies coalescent and Bayesian concordance analyses resolve early divergences of the grape family (Vitaceae). Cladistics.2018;34: 57-77.

61. Ren H, Lu LM,Soejima A, Luke Q, Zhang DX, Chen ZD, et al. Phylogenetic analysis of the grape family (Vitaceae) based on the noncoding plastid trnC-petN, trnH-psbA, andtrnL-F Taxon. 2011;60: 629-37.

62. Soejima A,Wen J. Phylogenetic analysis of the grape family (Vitaceae) based on three chloroplast markers. American Journal of Botany2006;93: 278-87.

63. Wen J,XiongZQ,NieZL, Mao LK, Zhu YB, Kan XZ,et al. Transcriptome sequences resolve deep relationships of the grape family. PLoS One.2013; 8: e74394.

64. Zhang N, Wen J, Zimmer EA. Congruent deep relationships in the grape family (Vitaceae) based on sequences of chloroplast genomes and mitochondrial genes via genome skimming. PLoS One.2015;10: e0144701.

65. Chu ZF, Wen J, Yang YP, NieZL, Meng Y. Genome size variation and evolution in the grape family Vitaceae. Journal of Systematics and Evolution.2018;56: 273-82.

66. Habib S, Dang VC,Ickert-Bond SM, Zhang JL, Lu LM, Wen J,et al. Robust Phylogeny of Tetrastigma(Vitaceae) Based on Ten Plastid DNA Regions: Implications for Infrageneric Classification and Seed Character Evolution. Front Plant Sci.2017;26: 590.

67. Chen PT, Wen J, Chen LQ. Spatial and temporal diversification of Tetrastigma(Vitaceae). gardens bulletin singapore2011;63: 313-3.

68. Lu,LM, Wang, W, Chen,ZD, Wen, J. Phylogeny of the non-monophyletic Cayratia Juss. (Vitaceae) and implications for character evolution and biogeography. Mol Phylogenet. Evol. 2013;68: 502-5.

69. Wang QR, Huang ZR, Gao CS, Ge YQ, Cheng, RB. The complete chloroplast genome sequence of Rubus hirsutusThunb. and a comparative analysis within Rubus species.Genetica.2021;149: 5-6.

70. Li X, Yang Y, Henry RJ,Rossetto M, Wang YT, Chen SL. Plant DNA barcoding: from gene to genome. Biol Rev. 2015;90: 157-66.

71. Islam SU, Dar T,Khuroo AA, Bhat BA, Malik AH. DNA barcoding aids in identification of adulterants of trillium govanianum Wall. ex D. Don. J Appl Res Med Aroma. 2021;23: 100305.

72. Neubig KM, Whitten WM,Carlsward BS, Blanco MA,Endara L, Williams NH,et al. Phylogenetic utility of ycf1 in orchids: a plastid gene more variable than matk. Plant Systematics \& Evolution.2009;277: 75-84.

73. Jiao LC, Yu M,Wiedenhoeft AC, He T, Li JN, Liu B,Jiang XM,et al. DNA Barcode Authentication and Library Development for the Wood of Six Commercial Pterocarpus Species: the Critical Role of Xylarium Specimens. Sci Rep. 2018; 8: 1945.

74. Jiao, LC, Lu, Y, He, T, Li, JN, Yin, YF. A strategy for developing high-resolution DNA barcodes for species discrimination of wood specimens using the complete chloroplast genome of three Pterocarpus species. Planta2019, 250, 94-104. 10.1007/s00425-019-03150-1.

75. Li YT, Dong Y, Liu YC, Yu XY, Yang MS,Huang YR. Comparative Analyses of Euonymus Chloroplast Genomes: Genetic Structure, Screening for Loci With Suitable Polymorphism, Positive Selection Genes, and Phylogenetic Relationships Within Celastrineae. Frontiers in Plant Science.2021;11: 593984.

76. Li QJ,Su N, Zhang L, Tong RC, Zhang XH, Wang JR, et al. Chloroplast genomes elucidate diversity, phylogeny, and taxonomy of Pulsatilla(Ranunculaceae). Sci Rep. 2020;10: 19781.

77. Zhao DN, Ren Y, Zhang JQ. Conservation and innovation: plastome evolution during rapid radiation of Rhodiola on the Qinghai-Tibetan Plateau. Mol PhylogenetEvol. 2019;144: 106713.

78. Gao CM, Deng YF,Wang J. The Complete Chloroplast Genomes of Echinacanthus Species (Acanthaceae): Phylogenetic Relationships, Adaptive Evolution, and Screening of Molecular Markers. Front Plant Sci. 2018;9: 1989.

79. Zhang L, Wang S,Su C, Harris AJ, Zhao L,Su N, et al. Comparative Chloroplast Genomics and Phylogenetic Analysis of Zygophyllum(Zygophyllaceae) of China. Front Plant Sci. 2021;12: 723622.

80. Tröndle D,Schröder S,Kassemeyer HH, Kiefer C, Koch MA, Nick P. Molecular phylogeny of the genus Vitis (Vitaceae) based on plastid markers. Am J Bot. 2010;97.

81. Chen Q, Wu XB,Zhang D. Comparison of the abilities of universal, super, and specific DNA barcodes to discriminate among the original species of Fritillariaecirrhosaebulbus and its adulterants. PLoS One. 2020; 15.

82. Xu WB, Xia BS, Li XW. The complete chloroplast genome sequences of five pinnate-leaved Primula species and phylogenetic analyses. Sci Rep.2020;10: 20782.

83. Yang XY, Liu YL, HouAJ, Yang Y, Tian X, He LY. Systematic review for geo-authenticLoniceraeJaponicae Front Med. $2017 ; 11: 203-13$. 
84. Yang JY, Pak JH, Maki M,Kim SC. Multiple origins and the population genetic structure of Rubus takesimensis (Rosaceae) on Ulleung Island: Implications for the genetic consequences of anagenetic speciation. PLoS One.2019;14: e0222707.

85. Wang AS, Wu HW, Zhu XC, Lin JM. Species Identification of Conyza bonariensisAssisted by Chloroplast Genome Sequencing. Front Genet. 2018;9: 374.

86. Zhou HT, Hu SL, Guo BL, Feng XF, Yan YN, Li JS. A study on genetic variation between wild and cultivated populations of Paeonia lactiflora Yao xuexue bao = Acta pharmaceuticaSinica. 2002;37: 383-8.

87. Zhang SB, Li C, Gu W,QiuRL, Chao JG, Pei LF, et al. Metabolomics analysis of dandelions from different geographical regions in China. Phytochemical Analysis. 2021; 32: 899-906.

88. Guo J, Yuan YH, Dou P, Yue TL. Multivariate statistical analysis of the polyphenolic constituents in kiwifruit juices to trace fruit varieties and geographical origins. Food Chemistry.2017;232: 552-9.

89. Miao LL, Zhou QM, Peng C, Meng CW, Wang XY,Xiong L. Discrimination of the Geographical Origin of the Lateral Roots of Aconitum carmichaelii Using the Fingerprint, Multicomponent Quantification, and Chemometric Methods. Molecules. 2019;24: 4124.

90. Long WJ, Wu HL, Wang T, Dong MY, Chen LZ,Yu RQ. Fast identification of the geographical origin of Gastrodiaelata using excitation-emission matrix fluorescence and chemometric methods. Spectrochim Acta A Mol BiomolSpectrosc.2021;258: 119798.

91. Guo, Guo, Zhao, Hou.Newly developed SSR markers reveal genetic diversity and geographical clustering in Paeonia suffruticosa based on flower colour. The Journal of Horticultural Science and Biotechnology.2018;93:416-24.

92. Zhang YC, Ge M, Wang XH, Zeng Y, Zeng Z. Discrimination of Moutan Cortex from different sources and geographical origins for quality evaluation using microsatellite markers coupled with chemical analysis. Biochemical Systematics and Ecology.2020;89: 104005.

93. Kumar V, Roy BK.Population authentication of the traditional medicinal plant Cassia tora based on ISSR markers and FTIR analysis. Scientific reports.2018;8.

94. Huang Z, Hu YY, Wang QQ, ChenKR. Pharmacognosy differentiation of Radix Tetrastigmae from Zhejiang and Guangxi. J Zhejiang Chin Med Univ. 2007;31: 759-60.

95. Li SM, Li Q, Sun CL, Peng X. Comparative study on multiple chemical pattern recognition combined with fingerprint of Tetrastigmahemsleyanumfrom different habitats. Chinese Traditional and Herbal Drugs.2020;51: 197-203.

96. Jiao, CW., Xu,ZP,Bian, QW, Forsberg, E, Tan, Q,Peng, X,He, SL. Machine learning classification of origins and varieties of Tetrastigmahemsleyanumusing a dual-mode microscopic hyperspectral imager.Spectrochimica Acta Part A: Molecular and Biomolecular Spectroscopy.2021;261: 120054.

97. Zhou DR, Yu Y, Hu RW, Li ZM. Discrimination of Tetrastigmahemsleyanumaccording to geographical origin by near-infrared spectroscopy combined with a deep learning approach. Spectrochim Acta A Mol BiomolSpectrosc.2020;238: 118380.

98. Wang YH, Jiang WM, Ye WQ, Fu CX,GitzendannerM.A, Soltis PS,et al. Evolutionary insights from comparative transcriptome and transcriptomewide coalescence analyses in Tetrastigmahemsleyanum. BMC Plant Biol.2018;18: 208.

99. Yin MH, Zhan XL, Xu WH, Xie NN, Cai H, Chen RH. Genetic diversity analysis of Tetrastigmahemsleyanum Diels et Gilg germplasm resources by random amplified polymorphic DNA technique. Acta Agric Zhejiangensis.2018; 30: 1839-48.

100. Yin MH, Shi GY, Yu XT, Chen MG, Liao Y, Wan ZT. Genetic diversity analysis of Tetrastigmahemsleyanum Diels et Gilg germplasm accessions by SRAP markers.J Plant Genet Resour.2018; 19: 1222-8.

101. Plant Working Group CBOL. A DNA barcode for land plants. Proc Natl Acad Sci. 2009;106: 12794-7.

102. Lee SR, Kim K, Lee BY, Lim CE. Complete chloroplast genomes of all six Hosta species occurring in Korea: molecular structures, comparative, and phylogenetic analyses. BMC Genomics.2019;20: 833.

103. Yang JJ, Zhang J, Han RX, Zhang F,Mao AJ, Luo J,et al. Target SSR-Seq: a novel SSR genotyping technology associate with perfect SSRs in genetic analysis of cucumber varieties. Front Plant Sci.2019;10:531.

104. Zhu M, Feng PP, Ping JY, Li JY,Su YJ,Wang T. Phylogenetic significance of the characteristics of simple sequence repeats at the genus level based on the complete chloroplast genome sequences of Cyatheaceae. Ecology and Evolution2021;11: 14327-40.

105. Song SL, Lim PE,Phang SM, Lee WW, Hong DD,Prathep A. Development of chloroplast simple sequence repeats (cpSSRs) for the intraspecific study of Gracilariatenuistipitata (Gracilariales, Rhodophyta) from different populations. BMC Res Notes.2014;7: 77.

106. Kapil A, RaiPK,Shanker A. ChloroSSRdb: a repository of perfect and imperfect chloroplastic simple sequence repeats (cpSSRs) of green plants. Database: the journal of biological databases and curation. 2014;107.10.1093/database/bau107.

107. Zhu B, Hua JW, Liu K, Ji QY, Wu JF, Qi C. ISSR analysis of genetic diversity of Tetrastigma hemsleyanum, a rare Chinese medicinal herb.Acta Agric Univ Jiangxiensis.2015;37: 914-9.

108. Doyle JJ. A rapid DNA isolation procedure for small quantities of fresh leaf tissue. Phytochem Bull. 1987;19: 11-5.

109. Tillich M,Lehwark P,Pellizzer T, Ulbricht-Jones ES, Fischer A, Bock R,et al. GeSeq - versatile and accurate annotation of organelle genomes. Nucleic Acids Res. 2017; 45: W6-W11.

110. Shi LC, ChenHM, Jiang M, Wang LQ, Wu X, Huang LF, et al. CPGAVAS2, an integrated plastome sequence annotator and analyzer. Nucleic Acids Res. 2019;47: W65-W73.

Page 22/24 
111. Greiner S,Lehwark P, Bock R. OrganellarGenomeDRAW (OGDRAW) version 1.3.1: expanded toolkit for the graphical visualization of organellar genomes.Nucleic Acids Res.2019;47: W59-W64.

112. Kumar S,Stecher G, Tamura K. MEGA7: Molecular Evolutionary Genetics Analysis Version 7.0 for Bigger Datasets. Mol Biol Evol.2016;33: 1870-4.

113. Sharp PM, Li WH. The codon Adaptation Index-a measure of directional synonymous codon usage bias, and its potential applications. Nucleic Acids Res.1987;15: 1281-95.

114. Ali A, Jaakko H, Peter P. Irscope: an online program to visualize the junction sites of chloroplast genomes. Bioinformatics $2018,17,3030-1$.

115. Kurtz S, Schleiermacher C. REPuter: fast computation of maximal repeats in complete genomes. Bioinformatics 1999;15: 426-7.

116. Beier S, Thiel T,Münch T, Scholz U,Mascher M. MISA-web: a web server for microsatellite prediction. Bioinformatics 2017;33: 2583-5.

117. Mower JP. The PREP suite: predictive RNA editors for plant mitochondrial genes, chloroplast genes and user-defined alignments.Nucleic Acids Res. 2009;37: W253-9.

118. Katoh K,Standley DM. MAFFT multiple sequence alignment software version 7: improvements in performance and usability. Mol Biol Evol. 2013;30: 772-80.

119. Rozas J, Ferrer-Mata A, Sánchez-DelBarrio JC,Guirao-Rico S,Librado P, Ramos-Onsins, SE, et al.DnaSP 6: DNA Sequence Polymorphism Analysis of Large Data Sets. Mol Biol Evol. 2017;34: 3299-302.

120. Hall TA. BioEdit: A User-Friendly Biological Sequence Alignment Editor and Analysis Program for Windows 95/98/NT. Nucleic Acids Symposium Series. 1999; 41: 95-8.

\section{Figures}

\section{Figure 1}

The geographical distributions and morphological characteristics of Tetrastigma hemsleyanum from different regions. (A) Regional distribution map of Tetrastigma hemsleyanum sample collection (B) The microscopic characters of the dried roots from Tetrastigma hemsleyanum: 1. brown patches; 2. marginal orifice catheter; 3. cluster crystals of calcium oxalate; 4 . needle crystals of calcium oxalate; 5 . starch granules; 6 . cork cell. (C) Comparison of macroscopic characters of the root tubers of Tetrastigma hemsleyanum from five different regions.

\section{Figure 2}

The chloroplast genome map of Tetrastigma hemsleyanum from five different regions. Genes labeled inside the circle are transcribed clockwise, while those outside the circle are transcribed anti-clockwise. The tick lines exhibited the extent of the Inverted Repeats (IRA and IRB) separating the Large Single-Copy (LSC) and the Small Single-Copy (SSC) regions. The darker gray and the lighter gray in the inner circle corresponds to GC and AT content, respectively. Genes with different functions are represented in different colors.

\section{Figure 3}

Comparison of the LSC, IR, and SSC junction regions among five Tetrastigma hemsleyanum samples with different geographical origins, Tetrastigma planicaule, Ampelopsis japonica and Vitis vinifera cp genomes.

\section{Figure 4}

Analysis of simple sequence repeats (SSRs) in Vitaceae plants and Tetrastigma hemsleyanum species with different geographical origins. (A) The number of different types of SSRs in five samples of Tetrastigma hemsleyanum from different regions.(B) The number of different types of SSRs in the cp genomes of Tetrastigma hemsleyanum sample from Jiangxi Province, Tetrastigma planicaule, Ampelopsis japonica and Vitis vinifera.

\section{Figure 5}

Comparison of potential mutational hotspots in the complete chloroplast genomes among Vitaceae plants and Tetrastigma hemsleyanum samples from different regions. (A) Nucleotide diversity (Pi) analysis among four Vitaceae chloroplast genomes. (B) Nucleotide diversity (Pi) analysis in the cp 
genomes of Tetrastigma hemsleyanum from five different regions. Sliding window length was $800 \mathrm{bp}$ and step size was selected as $200 \mathrm{bp}$. The Pi value of each window is shown on the $Y$-axis, and their positions of the midpoint represented on the $X$-axis.

\section{Figure 6}

Phylogenetic relationships based on the conserved chloroplast protein coding genes from five samples of Tetrastigma hemsleyanum and other representative Vitaceae species. (A) The tree was constructed using maximum likelihood (ML) method. (B) The tree was constructed using maximum parsimony (MP) method. The number above each node referred to the bootstrap value from 500 replicates. The areas corresponding to blue, lavender and yellow represented the tribes of Viteae, Ampelopsideae and Cayratieae, respectively. Melaleuca alternifolia and Melaleuca cajuputi were set as the outgroups for phylogenetic analysis

\section{Figure 7}

Comparative analysis of $t r n L$ and $t r n N$ sequences of $T$. hemsleyanum samples (A) Agarose gel electrophoresis of PCR products of five DNA barcodes from T. hemleyanum in Zhejiang Province. (B) Genetic distance analysis between the samples of T. hemsleyanum in Jiangxi Province and other regions, three representative Vitaceae species as well as two Melaleuca species based on $t r n L$ sequence, (C) $t r n N$ sequence and (D) combination of $t r n L+t r n N$ sequences. The Neighbor-Joining (NJ) trees of 21 samples of $T$. hemsleyanum from different regions and 3 representative Vitaceae species, based on $\operatorname{trn} L$ sequence $(\mathrm{E})$ and $t r n N$ sequence $(\mathrm{F})$.

\section{Figure 8}

The Neighbor-Joining (NJ) tree analysis of $21 \mathrm{~T}$. hemsleyanum samples from different regions and 3 representative Vitaceae species, based on the combination of $t r n L+t r n N$ sequence. The six colors represent samples of $T$. hemsleyanum from six different provinces.

\section{Supplementary Files}

This is a list of supplementary files associated with this preprint. Click to download.

- SupplementaryFigures.docx

- SupplementaryTables.docx 